\title{
Optical vortices 30 years on: OAM manipulation from topological charge to multiple singularities
}

\author{
Yijie Shen $\mathbb{1}^{1,2}$, Xuejiao Wang ${ }^{3}$, Zhenwei Xie (1) ${ }^{4}$, Changjun Min ${ }^{4}$, Xing Fu (1,2, Qiang Liu ${ }^{1,2}$, Mali Gong ${ }^{1,2}$ and \\ Xiaocong Yuan (10)
}

\begin{abstract}
Thirty years ago, Coullet et al. proposed that a special optical field exists in laser cavities bearing some analogy with the superfluid vortex. Since then, optical vortices have been widely studied, inspired by the hydrodynamics sharing similar mathematics. Akin to a fluid vortex with a central flow singularity, an optical vortex beam has a phase singularity with a certain topological charge, giving rise to a hollow intensity distribution. Such a beam with helical phase fronts and orbital angular momentum reveals a subtle connection between macroscopic physical optics and microscopic quantum optics. These amazing properties provide a new understanding of a wide range of optical and physical phenomena, including twisting photons, spin-orbital interactions, Bose-Einstein condensates, etc., while the associated technologies for manipulating optical vortices have become increasingly tunable and flexible. Hitherto, owing to these salient properties and optical manipulation technologies, tunable vortex beams have engendered tremendous advanced applications such as optical tweezers, high-order quantum entanglement, and nonlinear optics. This article reviews the recent progress in tunable vortex technologies along with their advanced applications.
\end{abstract}

\section{Introduction}

Vortices are common phenomena that widely exist in nature, from quantum vortices in liquid nitrogen to ocean circulation and typhoon vortices and even to spiral galaxies in the Milky Way, manifesting themselves not only in macroscopic matter but also in structured electromagnetic and optical fields. This year is the 30th anniversary of the birth of optical vortices (OVs). In 1989, Coullet et al. ${ }^{1}$ found the vortex solutions of the MaxwellBloch equations and created the concept of OVs, inspired by hydrodynamic vortices. Before the proposal of OVs, the analogy between laser physics and fluids/superfluids was already recognized ${ }^{2}$ as early as 1970 by reducing the laser equations to complex Ginzburg-Landau equations (CGLEs), which constitute a class of universal models

\footnotetext{
Correspondence: Xing Fu (fuxing@mail.tsinghua.edu.cn) or

Xiaocong Yuan (xcyuan@szu.edu.cn)

${ }^{1}$ Key Laboratory of Photonic Control Technology (Tsinghua University), Ministry of Education, 100084 Beijing, China

${ }^{2}$ State Key Laboratory of Precision Measurement Technology and Instruments, Department of Precision Instrument, Tsinghua University, 100084 Beijing, China Full list of author information is available at the end of the article.
}

describing pattern formation in a vast variety of phenomena such as superconductivity, superfluidity, and Bose-Einstein condensation ${ }^{3}$. Later, many hydrodynamic features, such as chaos, multistability, and turbulence, were analogically studied in optical fields ${ }^{4-6}$ and observed in laser systems ${ }^{7-9}$. Among the various hydrodynamic effects, the vortex soliton is quite attractive due to its distinctive structure carrying a singularity ${ }^{5-7}$. Analogous to the flow singularity in a fluid vortex, an optical vortex soliton has a phase singularity that appears as an isolated dark spot possessing the topological charges (TCs) of a helical phase $\mathrm{s}^{5,10}$. Novel optical vortex solitons were intensively explored based on CGLEs. For instance, stable vortex solitons ${ }^{11}$ and dissipative vortex solitons with trapping potentials ${ }^{12}$ can be solved by two-dimensional CGLEs. Topologically multicharged rotating vortex solitons ${ }^{13}$ and vortex excitation with feedback ${ }^{14}$ in lasers were also studied by CGLEs. Moreover, complicated threedimensional toroidal dissipative vortex solitons ${ }^{15}$ can also be characterized by CGLEs with high-order nonlinearity. In 1992, Allen et al. ${ }^{16}$ proposed the orbital angular

\section{(c) The Author(s) 2019}

(c) (i) Open Access This article is licensed under a Creative Commons Attribution 4.0 International License, which permits use, sharing, adaptation, distribution and reproduction c. in any medium or format, as long as you give appropriate credit to the original author(s) and the source, provide a link to the Creative Commons license, and indicate if changes were made. The images or other third party material in this article are included in the article's Creative Commons license, unless indicated otherwise in a credit line to the material. If material is not included in the article's Creative Commons license and your intended use is not permitted by statutory regulation or exceeds the permitted use, you will need to obtain permission directly from the copyright holder. To view a copy of this license, visit http://creativecommons.org/licenses/by/4.0/. 
momentum (OAM) in vortex beams (VBs) where the OVs propagate in paraxial beams, which unveiled a new understanding of the connection between macroscopic optics and quantum effects. As a typical representative of OVs, a VB has become a classical tool to study the properties of OVs because its generation can be easily realized in the laboratory ${ }^{17}$. VBs characterized by Hilbert factor $\exp (i \ell \theta)$, e.g., the Laguerre-Gaussian (LG) modes, can carry OAM equivalent to $\ell \hbar$ per photon $(\ell$ is an integer number), and this angular momentum (AM) can be much greater than the spin angular momentum (SAM) related to the photon $\operatorname{spin}^{10}$. The general results of these investigations created a new chapter of modern optics, i.e., singular optics ${ }^{18}$, which is a great leap forward in the development of traditional optics.

In the first 10 years, 1989-1999, the studies on OVs mainly focused on establishing fundamental theories and exploring basic physical phenomena, paving the way for further studies of the light-matter interaction, topological structures, and quantum nature of light. For instance, the dynamics of transverse pattern formation ${ }^{5,6}$, the interaction and OAM transfer between OVs and particles ${ }^{19-21}$, vortex solitons in a nonlinear medium ${ }^{22-24}$, nonlinear OAM-frequency transformation ${ }^{25,26}$, the topological phase in $\mathrm{OVs}^{27}$, the rotational Doppler effect ${ }^{28}$, and multisingularity arrays or vortex crystals ${ }^{5,29}$ were thoroughly studied. These novel theories lay the foundation for extending further widespread applications by using the unique properties of OVs.

In the second 10 years, 1999-2009, with the development of OAM manipulation, tremendous new applications rapidly emerged. In 2001, Zeilinger's group $^{30}$ realized the OAM-entangled photon pair, bringing OVs or twisted photons into quantum applications ${ }^{31}$. In 2002, Dholakia's group trapped particles with controlled rota$\operatorname{tion}^{32}$ and a three-dimensional structure ${ }^{33}$ by VBs, expanding the applications of optical tweezers ${ }^{34}$. In 2003, Harwit $^{35}$ demonstrated astrophysical OAM light generation and its applications in astrometry. In 2004, Zhuang ${ }^{36}$ used VBs as tweezers to assemble DNA biomolecules, opening up biomedical applications of OVs. In 2005, Ritsch-Marte's group ${ }^{37}$ used OAM in microscopy and imaging, and Tamburini et al. ${ }^{38}$ reported a superdiffraction-limit imaging approach using OAM. In 2008, Barreiro et al..$^{39}$ presented a coding technology using OAM, giving VBs great advantage for use in optical communications. During the decade, OVs were extended to almost every field of advanced optics.

In the last 10 years, 2009-2019, vortex and OAM applications have made many breakthroughs in rapid succession. In 2010, the optical lattice in far-field diffraction of OVs was unveiled as a very prompt and handy way to detect the $\mathrm{TC}^{40}$. In 2011, Capasso's group ${ }^{41}$ proposed the generalized laws of reflection and refraction, guiding $\mathrm{OV}$ generation in nanoscale metasurfaces. In 2012 , OAM beams were directly generated in a nanoscale emitter ${ }^{42}$. In 2013, Willner's group demonstrated terabitscale high-capacity optical communication via OAM multiplexing in both free space ${ }^{43}$ and fibres ${ }^{44}$. In 2016, Zeilinger's group ${ }^{45}$ generated extreme OAM states of over $10,000 \hbar$ and realized quantum entanglement of these states. In the recent three years, increasing numbers of tunable properties of OVs have been flexibly controlled at the nanoscale, including SAM-OAM conversion for classical $^{46}$ and quantum light ${ }^{47}$, tunable wavelength from visible $^{48,49}$ to $\mathrm{X}$-ray light ${ }^{50}$, ultra-broadband tunable $\mathrm{OAM}^{51}$, and tunable chirality ${ }^{52}$. Moreover, the timevarying OAM was recently revealed in extreme-ultraviolet VB with time-delay-tunable high harmonic generation ${ }^{53}$. To date, OVs have brought about numerous innovations in various fields and are still enabling great novelties with improved tunability.

Throughout the roadmap summarized above and depicted in Fig. 1, we can divide the 30-year development into three stages: the first 10 years, the fundamental theories stage; the second, the application development stage; and the third, the technology breakthrough stage. These three stages share one common theme of pursuing improved tunability of OVs because the realization of a broader tunable range of OVs can always benefit the birth of new applications. Thus, we propose the tunability of OVs as a better way to describe state-of-the-art achievements of OVs. Traditionally, tunable light always means that the wavelength can be tuned and sometimes means that the pulse width can be tuned for a pulsed laser; however, the tunability of OVs should be expanded to more dimensions due to their exotic properties. The tunability of OVs includes not only the spectral and temporal tunability but also the OAM-, chirality-, TC-, and singularity-distribution tunability. We present reviews in succession on the historical progress in the new tunable methods of OVs driven by the fundamental theories and then the numerous novel applications engendered by the improved tunability of OVs. In the "Properties of OVs" section, we review the fundamental theories and properties of OVs, providing a better understanding of the corresponding applications enabled by the unique properties. In "Progress in vortex generation, tuning, and manipulation" section, we review the generation and manipulation methods of OVs, developed from tuning the TC of a single singularity to controlling a multi-singularity array, including wavelength-, temporal-, and OAM-tuning technologies. In "Advanced applications of tunable VBs" section, we comprehensively review various advanced applications derived from vortex manipulation. Concluding remarks and prospects are given in the "Conclusions and perspectives" section. 


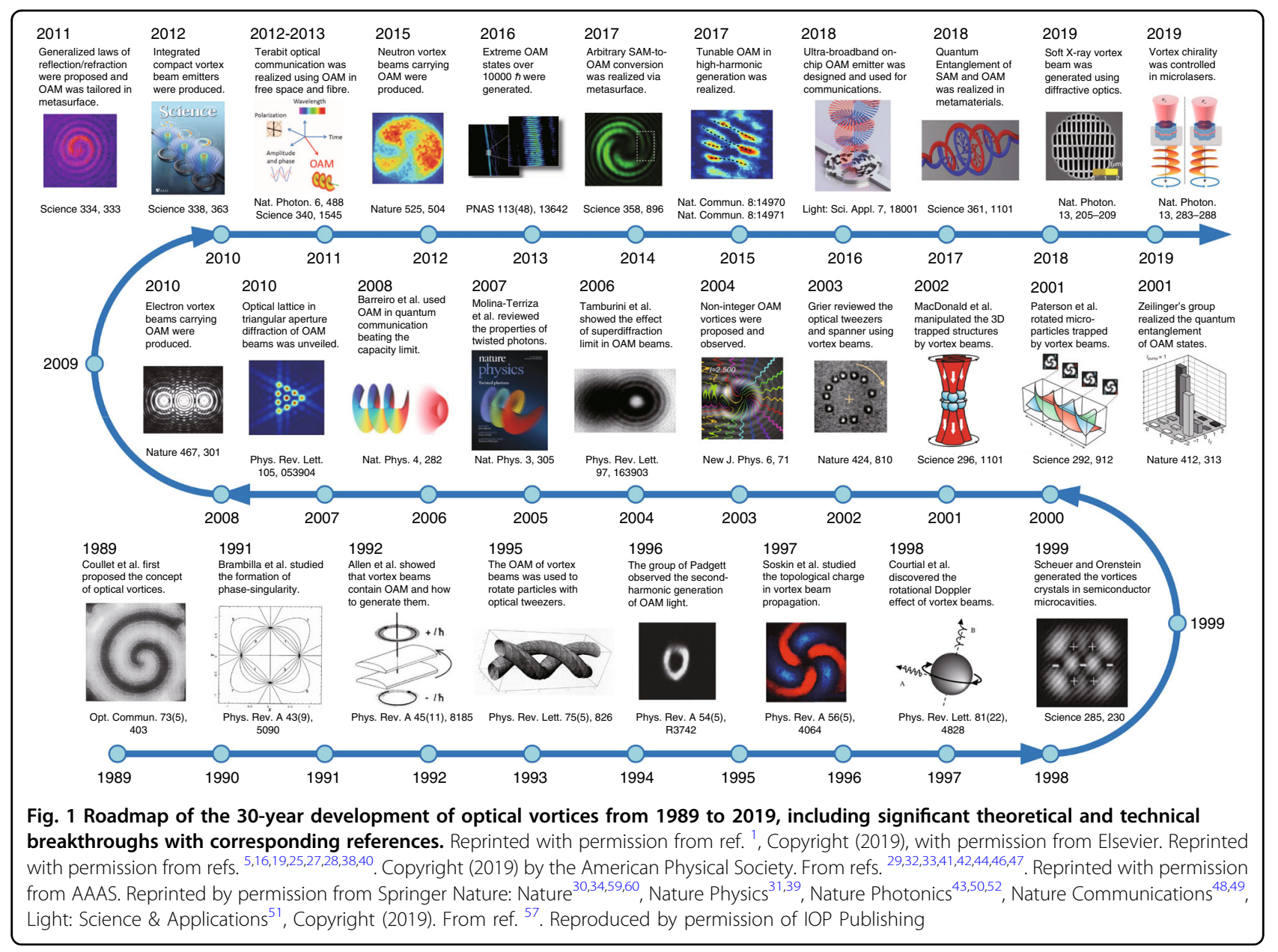

\section{Properties of OVs}

\section{Singularity and topological charge}

The salient properties of OVs are mostly related to the topological phase structure. Early in the 1970s before OVs were first observed, the topological structure in the wave phase was already under study. Nye and Berry ${ }^{54}$ demonstrated that wave trains with dislocations could induce a vortex structure where a singularity could be solved in the wave equation, which laid the foundation for the study of vortices in air, water, and even light waves, pushing the discovery of OVs. To understand the profound topology in a plain way, we can refer to a familiar artwork exhibiting a similar structure. Escher's painting Ascending and Descending shows an impossible scenario where the stairs are ascending clockwise yet have a seamless connection to their origin after a roundtrip, which is an artistic implementation of the Penrose Stairs ${ }^{55}$, as illustrated in Fig. 2. This structure is impossible in real space but possible in phase space. If the phase angle continually increases clockwise along a closed loop from 0 to $2 \pi \ell$ and returns to the origin, where the integer $\ell$ is called the TC, the angle zero is exactly equal to $2 \pi \ell$, forming a continuous phase distribution along the closed loop, similar to the topology of the well-known Möbius strip ${ }^{56}$. The centre spot of the closed loop where the phase cannot be defined is a phase singularity. The definition of the $\mathrm{TC}$ of a singularity for the phase distribution $\phi$ is given by:

$$
\ell=\frac{1}{2 \pi} \times \oint_{C} \nabla \phi(\mathbf{r}) \mathrm{d} r
$$

where $C$ is a tiny closed loop surrounding the singularity. For the light field with phase distribution $\exp (\mathrm{i} \ell \theta)$ carrying OAM of $\ell \hbar$ per photon, the TC of the centre phase singularity is $\ell$. The effect of TC is actually commonly seen in our daily life, e.g., the time distribution on earth has a singularity at the North Pole with a TC of $24 \mathrm{~h}$, the duration that the earth takes to rotate one cycle. The continuous phase along the closed loop results in an integer TC. However, as a peculiar case, a non-integer TC was also experimentally and theoretically investigated in $\mathrm{OVs}^{57,58}$. A phase singularity with a certain $\mathrm{TC}$ is a representation of a very simple vortex soliton yet acts as an important unit element in that more complex hydrodynamic vortices with chaos, attractors, and 
a

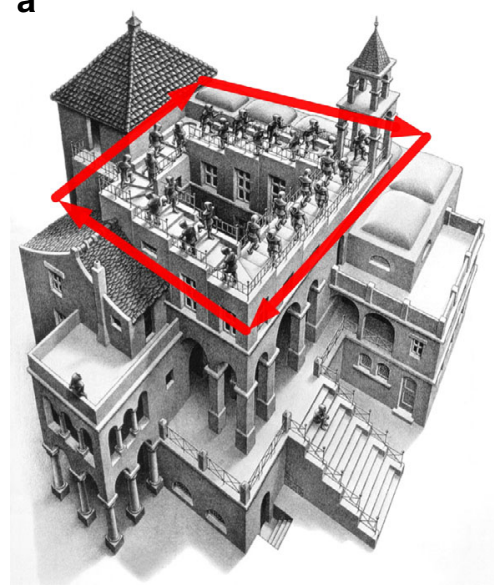

b

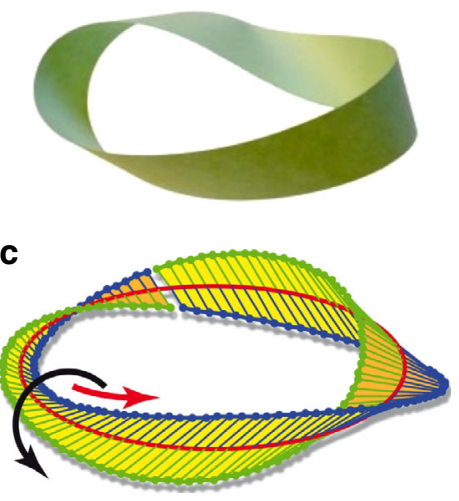

d

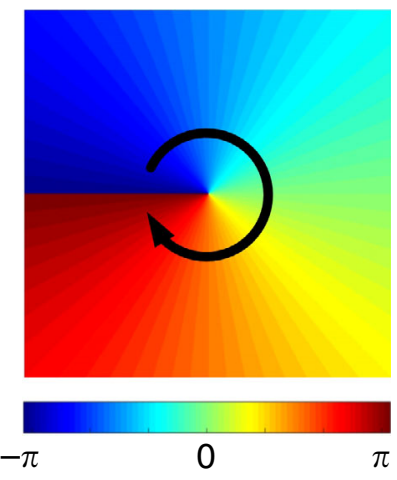

Fig. 2 Basic topological structure of vortex from art to science. The topological structures of $\mathbf{a}$ the Penrose Stair ${ }^{55}, \mathbf{b}, \mathbf{c}$ a Möbius strip ${ }^{56}$, and $\mathbf{d}$ the phase of a vortex soliton (Hilbert factor) are isomorphic, i.e., a physical value (displacement or angle) continually increases along a closed loop and coincides exactly with the origin after a roundtrip. $\mathbf{c}$ From ref. ${ }^{56}$. Reprinted with permission from AAAS

turbulence can be seen as the combination of a set of various singularities. This basic description is widely applicable to air $^{53}$, water ${ }^{4}$, light ${ }^{1}$, electron ${ }^{59}$, and neutron $^{60}$ vortex fields.

\section{Orbital angular momentum and vortex beams}

A VB is a paraxial light beam possessing Hilbert factor $\exp (\mathrm{i} \ell \theta)$ and carrying OVs along the propagation axis. OVs are not restricted to VBs, yet as typical OVs, VBs carrying OAM, also called OAM beams, are almost the most attractive form of OVs due to their unique quantum-classical-connection properties. There are already many review articles on OAM, especially on vortex generation $^{61,62}$, OAM on metasurfaces ${ }^{63}$, and basic OAM theories and applications ${ }^{64,65}$. However, few studies have focused on vortex tunability, which is the main theme of this article. For the introduction of basic theories of OAM, previous reviews usually used the well-known Poynting picture to describe the AM of the photon ${ }^{66,67}$, which leads to some difficulties, such as complex expressions of OAM and SAM, incompatibility with quantum optics, and the Abraham-Minkowski dilemma ${ }^{68}$. Here, we review the recently proposed new theory of the canonical picture ${ }^{69,70}$, which can overcome these difficulties, to introduce basic properties of OAM. The canonical momentum of light is represented as

$$
\mathbf{P}=\frac{g}{2} \operatorname{Im}\left[\tilde{\varepsilon} \mathbf{E}^{*} \cdot(\nabla) \mathbf{E}+\tilde{\mu} \mathbf{H}^{*} \cdot(\nabla) \mathbf{H}\right]
$$

where $\mathbf{H}$ is the magnetizing field. Gaussian units with $g=(8 \pi \omega)^{-1}, \tilde{\varepsilon}=\varepsilon+\omega \mathrm{d} \varepsilon / \mathrm{d} \omega$, and $\tilde{\mu}=\mu+\omega \mathrm{d} \mu / \mathrm{d} \omega$ are used. The canonical SAM and OAM densities are expressed as

$$
\mathbf{S}=\frac{g}{2} \operatorname{Im}\left[\tilde{\varepsilon} \mathbf{E}^{*} \times \mathbf{E}+\tilde{\mu} \mathbf{H}^{*} \times \mathbf{H}\right], \mathbf{L}=\mathbf{r} \times \mathbf{P}
$$

The total AM of light is $\mathbf{J}=\mathbf{S}+\mathbf{L}$. For a light beam, a rotating polarization leads to SAM, while a rotating wavefront leads to OAM. Consider a VB propagating along the $z$-axis:

$$
\mathbf{E}(r, \theta, z)=A(r, z) \frac{\hat{\mathbf{x}}+m \hat{\mathbf{y}}}{\sqrt{1+|m|^{2}}} \exp (i k z+i \ell \theta)
$$

The average SAM and OAM can be derived as ${ }^{69,70}$

$$
\frac{\mathbf{S}}{W}=\frac{\sigma \mathbf{k}}{\omega} \frac{\mathbf{L}}{\mathrm{W}}=\frac{\ell \mathbf{k}}{\omega} \frac{\mathbf{k}}{\mathrm{k}}
$$

where the power density $W=\frac{g \omega}{2}\left(\tilde{\varepsilon}|\mathbf{E}|^{2}+\tilde{\mu}|\mathbf{H}|^{2}\right)$ and $\sigma=\frac{2 \operatorname{Im}(m)}{1+|m|^{2}} . \sigma=+1(-1)$ and 0 correspond to left (right) circularly polarized light and linearly polarized light, respectively. Thus, Eq. (4) reveals that left (right) circularly polarized light carries an SAM of $+\hbar(-\hbar)$ per photon; the light with Hilbert factor $\exp (\mathrm{i} \ell \theta)$ carries an OAM of $\ell \hbar$ ( $\ell$ $=0, \pm 1, \pm 2, \ldots)$ per photon, where " \pm " reveals the chirality of the vortex, as demonstrated in Fig. 3. This is consistent with the AM quantization in quantum optics, i.e., the eigenvalues of SAM and OAM for the photon eigenstate yield $\hat{L}_{z}|\psi\rangle=\ell \hbar|\psi\rangle$ and $\hat{S}_{z}|\psi\rangle=\sigma \hbar|\psi\rangle$. Therefore, the phase factor $\exp (\mathrm{i} \ell \phi)$ provides a basic frame of VBs. 


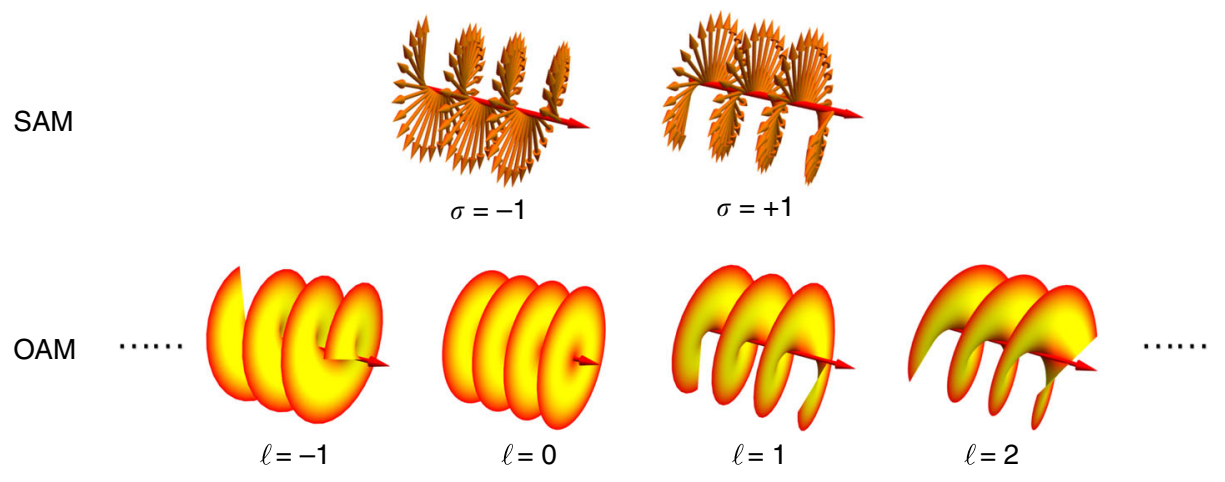

Fig. 3 The OAM of light beams is revealed by the phase distributions and the SAM by the polarizations. The phase distributions for various eigenstates of OAM and the polarizations (right- and left-handed circularly polarized) for the two eigenstates of SAM are illustrated
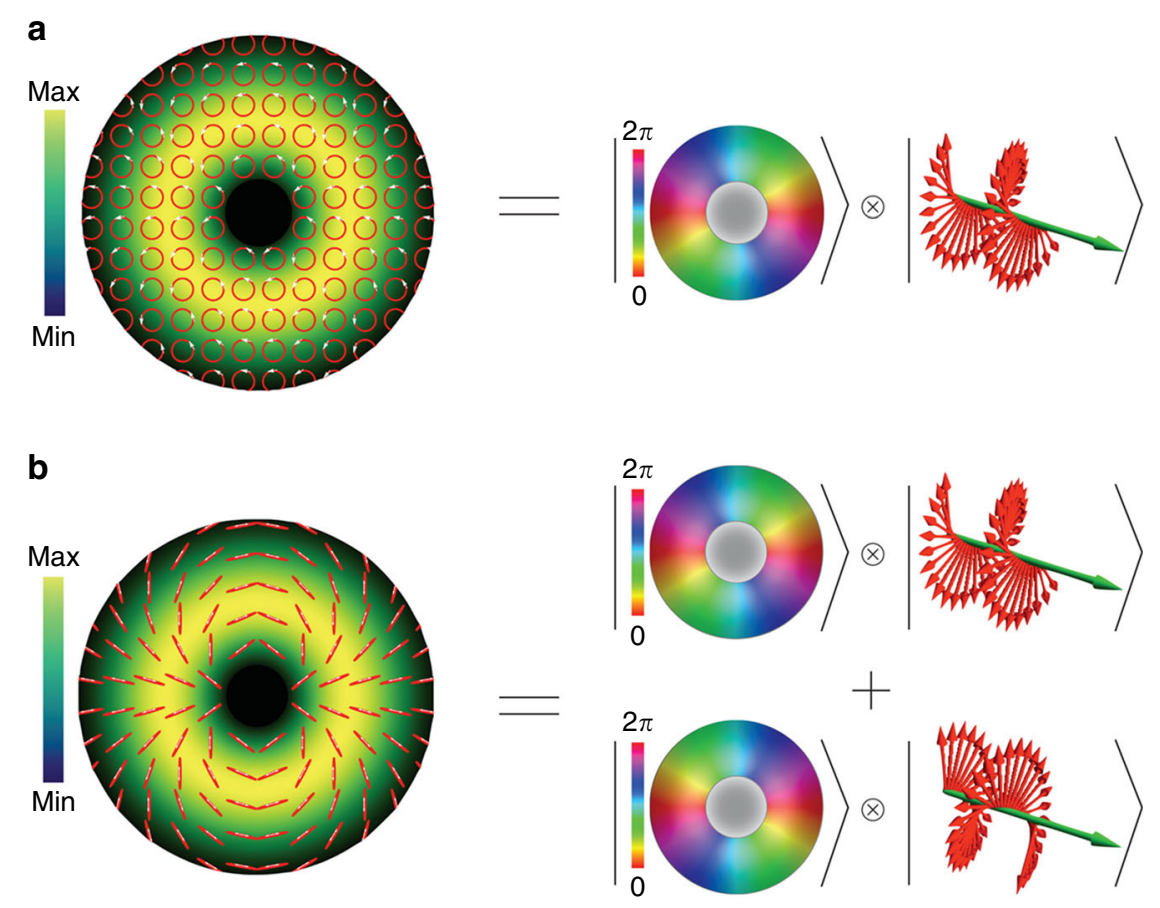

Fig. 4 Formation of vector beam with space-polarization nonseparability. a Circularly polarized OV with an azimuthally varying phase distribution. Such a state is considered separable, as it can be represented as the product of a spatially varying vortex phase and a polarization state vector. $\mathbf{b}$ Spider-like vector vortex represented as the superposition of the state of $\mathbf{a}$ with another state with the opposite phase variation and the opposite circular polarization. From ref. ${ }^{71}$. Reprinted with permission from AAAS

\section{Polarization and vector vortices}

The previous part focuses on the scalar light field, where the polarization is separable from the space. In scalar vortices, there are topological spatial phase structures, but the polarization is unchanged; e.g., Fig. 4a shows that a circularly polarized OV can be expressed as the product of a spatially varying vortex phase state and a circular polarization state ${ }^{71}$. If the polarization state has a spatially varying vector distribution forming vortex-like patterns, then the corresponding optical field is called polarization vortices or vector vortices, and the corresponding singularity is called a polarization singularity or a vector singularity $^{72,73}$. Based on the various topological disclinations of polarization, vector vortices can be categorized into many types, such as $\mathrm{C}$-point, $\mathrm{V}$-point, lemons, star, spider, and web, according to the actual vector morphology ${ }^{74}$. In contrast to the phase vortices carrying OAM, the vector vortices are always related to a complex SAM-OAM coupling; e.g., Fig. 4b shows a spider-like vector OV formed by the superposition of opposite phase 

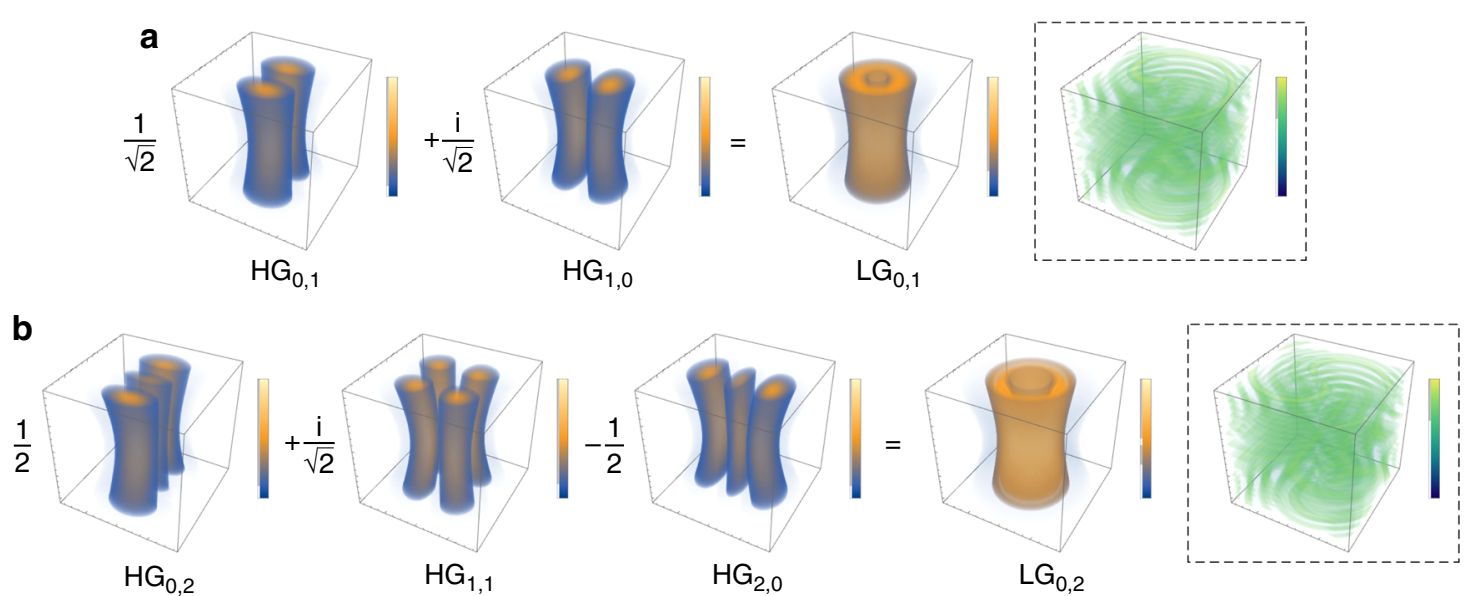

Fig. 5 Decomposition of $L G$ vortex beams. Examples of the decomposition of $L G$ modes $\left(L_{0,1}(\mathbf{a})\right.$ and $L G_{0,2}$ (b)) into $H G$ modes according to Eq. (7), where the insets in the dotted box show the corresponding vortex phase distributions

variations and opposite circular polarizations, where the total OAM is zero due to the sum of the two opposite phase variations but there is a complex SAM entangled with the space ${ }^{71}$.

\section{Classical models of OVs}

\section{LG and Hermite-Laguerre-Gaussian modes}

LG modes with circular symmetry are the earliest reported VBs carrying OAM ${ }^{16}$ and can be included in the general family of Hermite-Laguerre-Gaussian (HLG) modes with elliptical vortices ${ }^{75-77}$, thus accommodating the transform from the HG to LG mode, which has recently played increasingly important roles because the exploration of the more general structure of OVs always leads to novel applications:

$$
\begin{aligned}
\mathrm{HLG}_{n, m}(\mathbf{r}, z \mid \alpha)= & \frac{1}{\sqrt{2^{N-1} n ! m !}} \exp \left(-\pi \frac{\mid \mathbf{r}^{2}}{w}\right) \mathrm{HL}_{n, m}\left(\frac{\mathbf{r}}{\sqrt{\pi} w} \mid \alpha\right) \\
& \times \exp \left[\mathrm{i} k z+\mathrm{i} k \frac{r^{2}}{2 R}-\mathrm{i}(m+n+1) \vartheta\right]
\end{aligned}
$$

where $\mathrm{HL}_{n, m}(\cdot)$ is a Hermite-Laguerre (HL) polynomial ${ }^{75}$, $r=(x, y)^{\mathrm{T}}=(r \cos \phi, r \sin \phi)^{\mathrm{T}}, \quad R(z)=\left(z_{R}^{2}+z^{2}\right) / z$, $k w^{2}(z)=2\left(z_{R}^{2}+z^{2}\right) / z_{R}, \quad \vartheta(z)=\arctan \left(z / z_{R}\right)$, and $z_{R}$ is the Rayleigh range. For $\alpha=0$ or $\pi 2$, the $\mathrm{HLG}_{n, m}$ mode is reduced to the $\mathrm{HG}_{n, m}$ or $\mathrm{HG}_{m, n}$ mode. For $\alpha=\pi / 4$ or $3 \pi / 4$, the $\mathrm{HLG}_{n, m}$ mode is reduced to $\mathrm{LG}_{p, \pm \ell}$ mode $[p=\min (m, n), \ell=m-n]$. For the other interposed states, the HLG mode has multiple singularities with a total TC of $\ell$. As illustrated in Fig. 5, the $\mathrm{LG}_{p, \ell}$ mode can be decomposed into a set of Hermite-Gaussian (HG) $\operatorname{modes}^{16,17}$ :

$$
\mathrm{LG}_{p, \pm \ell}(x, y, z)=\sum_{K=0}^{m+n}( \pm \mathrm{i})^{K} b(n, m, K) \cdot \mathrm{HG}_{m+n-K, K}(x, y, z)
$$

$$
b(n, m, K)=\left.\left[\frac{(N-K) ! K !}{2^{N} n ! m !}\right]^{1 / 2} \frac{1}{K !} \frac{\mathrm{d}^{K}}{\mathrm{~d} t^{K}}\left[(1-t)^{n}(1+t)^{m}\right]\right|_{t=0}
$$

which also interprets the transformation to an $\mathrm{LG}_{p, \ell}$ mode from an $\mathrm{HG}_{n, m}$ mode through an astigmatic mode converter $(\mathrm{AMC})^{17}$.

\section{Helical-Ince-Gaussian and singularities hybrid evolution nature mode}

The Ince-Gaussian (IG) mode $^{78}$ is the eigenmode of the paraxial wave equation (PWE) separable in elliptical coordinates $(\xi, \eta)^{79}$ :

$$
\begin{aligned}
& \mathrm{IG}_{u, v}^{e, o}(x, y, z \mid \epsilon)= \\
& \frac{C^{e, o}}{w} I_{u, v}^{e, o}(\mathrm{i} \xi, \epsilon) I_{u, v}^{e, o}(\eta, \epsilon) \exp \left(-\frac{x^{2}+y^{2}}{w^{2}}\right) \\
& \exp \left[\mathrm{i} k z+\mathrm{i} k \frac{x^{2}+y^{2}}{2 R}-\mathrm{i}(u+1) \vartheta\right]
\end{aligned}
$$

where $C^{e, o}$ are normalization constants (the superscripts $e$ and $o$ refer to even and odd modes), $I_{u, v}^{e, o}(\cdot, \epsilon)$ are the even and odd Ince polynomials, with $0<v<u$ for even functions, $0<u<v$ for odd functions, and $(-1)^{u-v}=1$ for both, and $\epsilon \in(0, \infty)$ is the eccentricity. The special superposition of these modes can form a multi-singularity 
array with OAM, named the helical-IG (HIG) modes $^{80-82}$ :

$$
\mathrm{HIG}_{u, v}^{ \pm}(x, y, z \mid \epsilon)=\mathrm{IG}_{u, v}^{e}(x, y, z \mid \epsilon) \pm \mathrm{i} \cdot \mathrm{IG}_{u, v}^{o}(x, y, z \mid \epsilon)
$$

which carries multiple singularities with unit TC, having a total TC of $v$. Sharing the singularities hybrid evolution nature (SHEN) of the HIG and HLG modes, the SHEN mode is a very general family of structured Gaussian modes including the HG, LG, HLG, and HIG modes, the expression of which is ${ }^{83}$

$$
\begin{aligned}
& \operatorname{SHEN}_{n, m}(x, y, z \mid \beta, \gamma)=\sum_{K=0}^{N} \mathrm{e}^{\mathrm{i} \beta K} b(n, m, K) \\
& \cdot\left\{\begin{array}{c}
(-\mathrm{i})^{K} \mathrm{IG}_{N, N-K}^{e}\left(x, y, z \mid \epsilon=2 / \tan ^{2} \gamma\right), \text { for }(-1)^{K}=1 \\
(-\mathrm{i})^{K} \mathrm{IG}_{N, N-K+1}^{o}\left(x, y, z \mid \epsilon=2 / \tan ^{2} \gamma\right), \text { for }(-1)^{K} \neq 1
\end{array}\right.
\end{aligned}
$$

The SHEN mode is reduced to the HIG mode when $\beta=$ $\pm \pi / 2$, to the HLG mode when $\gamma=0$, to the HG mode when $(\beta, \gamma)=(0,0)$ or $(\pi, 0)$, and to the LG mode when $(\beta$, $\gamma)=( \pm \pi / 2,0)$. In addition, there is a graphical representation, the so-called SHEN sphere, to visualize the topological evolution of multi-singularity beams. Thus, the SHEN mode has great potential to characterize more general structure beams.

\section{Bessel and Mathieu modes}

Using the non-diffraction assumption in solving the PWE, we can also solve a set of eigenmodes. Under separable conditions in circular coordinates, the Bessel mode can be obtained as ${ }^{84}$

$$
\mathrm{B}_{\ell}(r, \theta, z)=J_{\ell}(\mu r) \exp (\mathrm{i} \ell \theta) \exp (\mathrm{i} k z)
$$

Bessel beams with $\ell \neq 0$ are VBs carrying $\ell \hbar$ OAM. Another non-diffraction solution separable in elliptical coordinates is the Mathieu modes ${ }^{85}$,

$$
\begin{aligned}
& \mathrm{M}_{m}^{e}(x, y, z \mid \epsilon)=C_{m} \mathrm{Je}_{m}(\xi, \epsilon) \mathrm{ce}_{m}(\eta, \epsilon) \exp \left(\mathrm{i} k_{z} z\right) \\
& \mathrm{M}_{m}^{o}(x, y, z \mid \epsilon)=S_{m} \mathrm{Jo}_{m}(\xi, \epsilon) \mathrm{se}_{m}(\eta, \epsilon) \exp \left(\mathrm{i} k_{z} z\right)
\end{aligned}
$$

where $C_{m}$ and $S_{m}$ are normalization constants, $\mathrm{Je}_{m}$ and $\mathrm{Jo}_{m}$ are radial Mathieu functions, and $\mathrm{ce}_{m}$ and $\mathrm{se}_{m}$ are angular Mathieu functions. Analogous to deriving the HIG mode, a helical Mathieu (HM) beam ${ }^{86}$ can carry multiple singularities and complex $\mathrm{OAM}^{87}$.

$$
\mathrm{HM}_{m}^{ \pm}(x, y, z \mid \epsilon)=\mathrm{M}_{m}^{e}(x, y, z \mid \epsilon) \pm \mathrm{i} \cdot \mathrm{M}_{m}^{o}(x, y, z \mid \epsilon)
$$

High-order Bessel and HM beams are often called nondiffractive VBs, whose unique properties have been extended to a great number of applications, such as particle assembly and optical communication ${ }^{88,89}$.

\section{SU(2) geometric modes}

When a resonator cavity fulfils the reentrant condition of a coupled quantum harmonic oscillator in SU(2) Lie algebra $^{90}$, the laser mode undergoes frequency degeneracy with a photon performing as an $\mathrm{SU}(2)$ quantum coherent state coupled with a classical periodic trajectory ${ }^{91}$, which is called an $\mathrm{SU}(2)$ geometric mode $(\mathrm{GM})^{92}$. The frequency degeneracy means that $\Delta f_{\mathrm{T}} / \Delta f_{\mathrm{L}}=P / Q=\Omega$ should be a simple rational number, where $P$ and $Q$ are two coprime integers, and $\Delta f_{\mathrm{T}}\left(\Delta f_{\mathrm{L}}\right)$ is the longitudinal (transverse) mode spacing. The wave-packet function of a planar GM is given by ${ }^{92}$

$$
\begin{aligned}
\Psi_{n_{0}}^{M}\left(x, y, z ; \phi_{0} \mid \Omega\right)= & \frac{1}{2^{M / 2}} \sum_{K=0}^{M} \sqrt{\frac{M !}{K !(M-K) !}} \\
& \cdot \mathrm{e}^{\mathrm{i} K \phi_{0}} \cdot \psi_{n_{0}+Q \cdot K, 0, s_{0}-P \cdot K}^{(\mathrm{HG})}(x, y, z)
\end{aligned}
$$

where phase $\phi_{0}$ is related to the classical periodic trajectory. $\psi_{n, m, s}^{(\mathrm{HG})}$ represents the $\mathrm{HG}_{n, m}$ mode considering the frequency-dependent wavenumber $k_{n, m, s}=2 \pi f_{n, m, s} / c$, where $f_{n, m, s}=s \cdot \Delta f_{\mathrm{L}}+(n+m+1) \cdot \Delta f_{\mathrm{T}}$. If the HG bases are transformed into LG bases, then the circular GM is obtained $^{92}$ :

$$
\begin{aligned}
\Phi_{n_{0}}^{M}\left(x, y, z ; \phi_{0} \mid \Omega\right)= & \frac{1}{2^{M / 2}} \sum_{K=0}^{M} \sqrt{\frac{M !}{K !(M-K) !}} \\
& \cdot \mathrm{e}^{\mathrm{i} K \phi_{0}} \cdot \psi_{0, \pm\left(n_{0}+Q \cdot K\right), s_{0}-P \cdot K}^{(\mathrm{LG})}(x, y, z)
\end{aligned}
$$

where $\psi_{p, \ell, s}^{(\mathrm{LG})}$ represents the $\mathrm{LG}_{p, \ell}$ mode considering the frequency-dependent wavenumber. The vortex circular GM has many unique properties, such as an exotic 3D structure, multiple singularities, and fractional $\mathrm{OAM}^{92,93}$. Note that there are other types of $\mathrm{SU}(2)$ modes related to OAM with special properties, such as Lissajous modes ${ }^{94}$, trochoidal modes ${ }^{95}$, polygonal $\mathrm{VBs}^{96}$, and $\mathrm{SU}(2)$ diffraction lattices ${ }^{97}$ as shown in Fig. 6d, e.

The above forms are classical VBs in free space, which are just optical modes carrying OAM. In addition, there are OVs that are formed by non-OAM beams, as reviewed in the following.

\section{Optical Möbius strips}

A direct idea is to arrange the optical parameter into the form of Möbius strips, one of the classical topological models. This type of OV is called an optical Möbius strip (OMS). A simple vortex phase with integer TC can be seen as a phase OMS. In addition to phase vortices, more OMSs can be obtained by arranging the polarization: the major and minor axes of the polarization ellipses that 


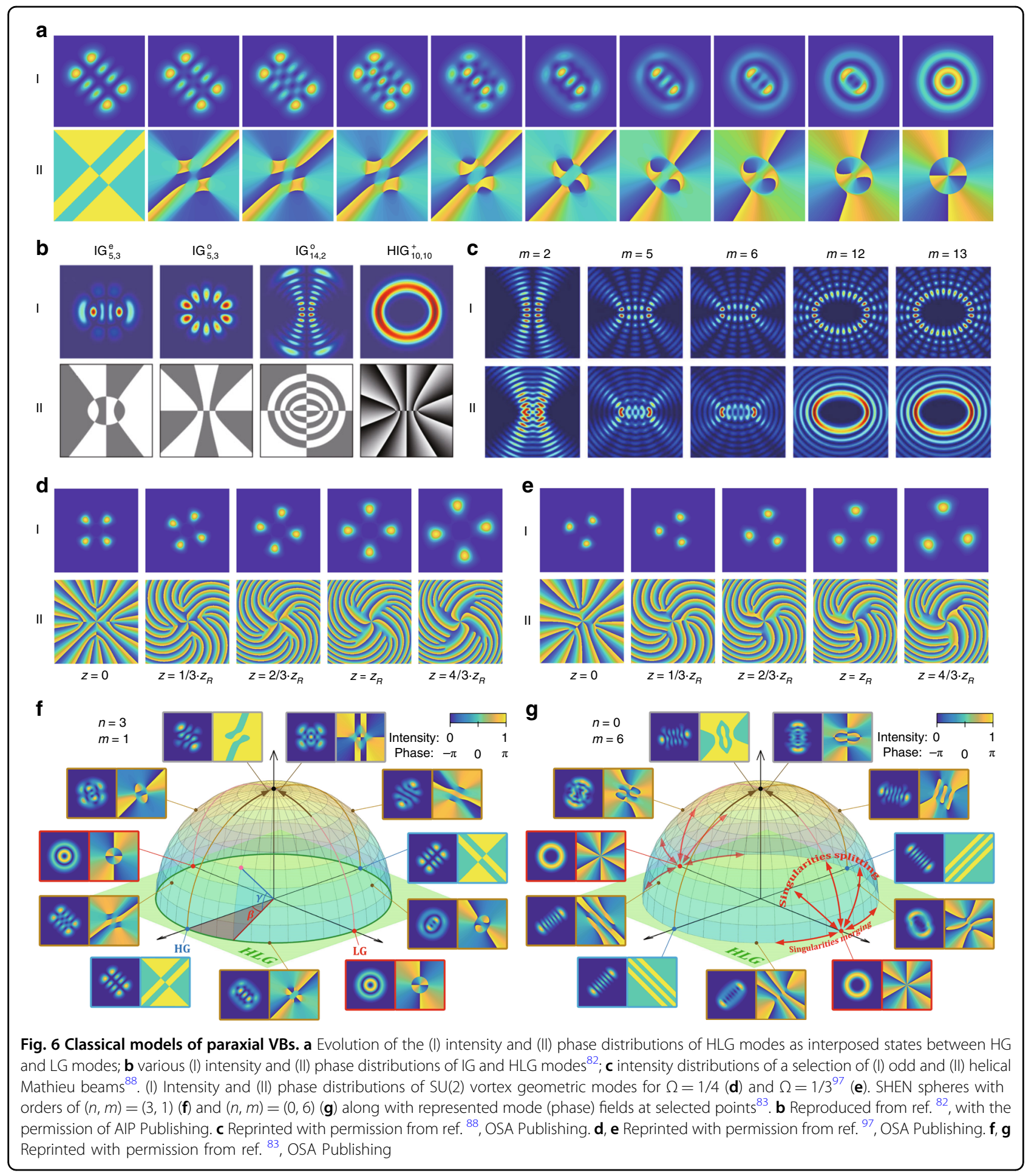

surround singular lines of circular polarization in threedimensional optical ellipse fields can be organized into an OMS, as theoretically proposed ${ }^{98,99}$ and experimentally observed $^{49}$. Currently, multitwist OMSs can be controlled in both paraxial and nonparaxial vector beams ${ }^{56,100}$. By combining other spatial and optical parameters into
OMSs, more complex structures, such as 3D solitons and topological knots, can be proposed for OVs ${ }^{101}$.

\section{Vortex knots}

The vortex core of an OV can not only be distributed along the propagation axis of a beam but also form 
$\mathbf{a}_{0}$

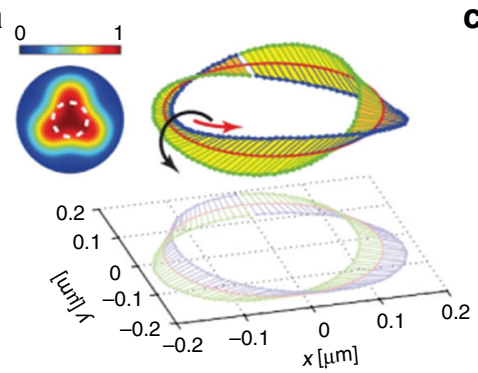

$\mathbf{b}_{0}$

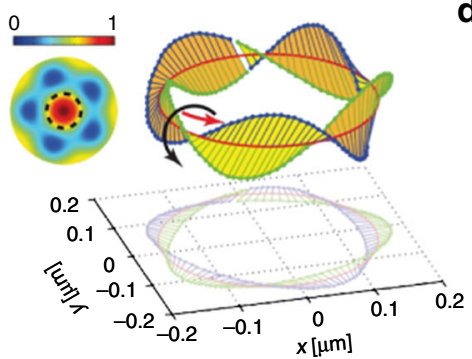

c

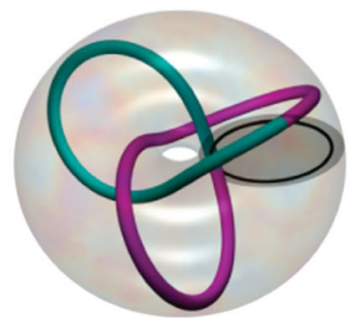

d
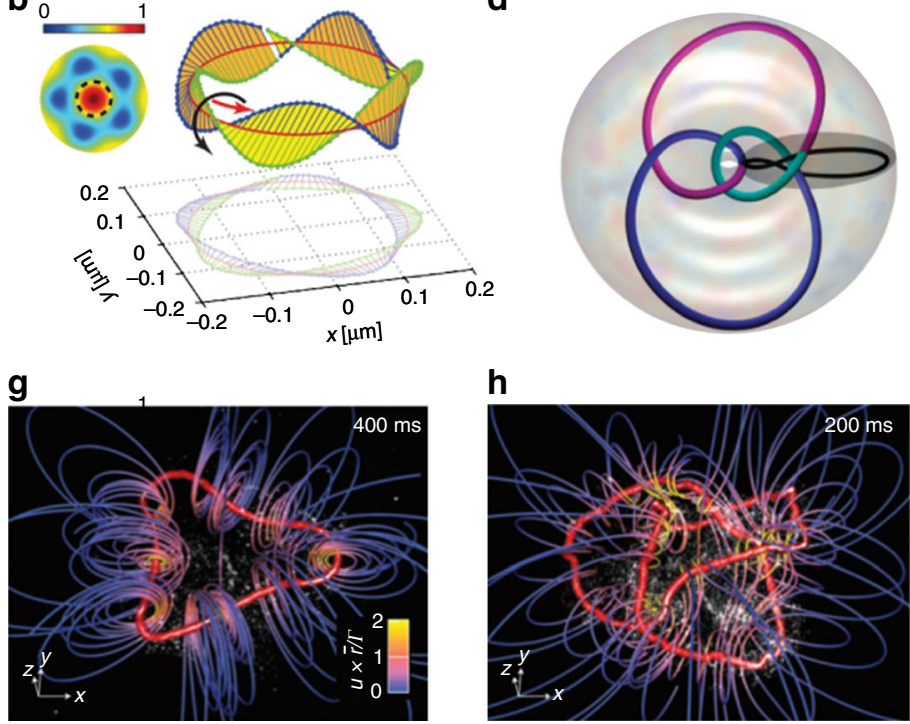

h

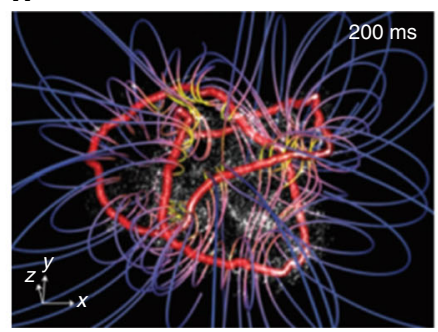

e

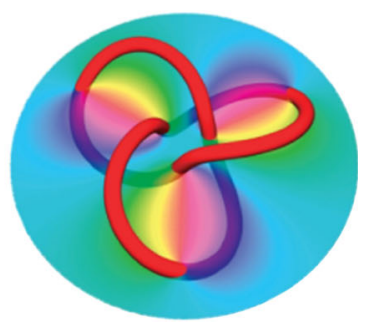

f

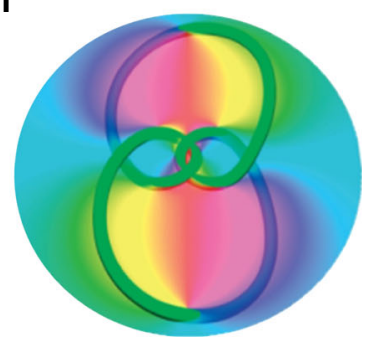

i

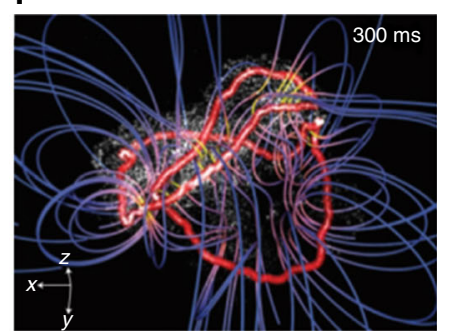

Fig. 7 Classical models of spatial nonparaxial OVs. Polarization topology of optical Möbius strips with twisted TCs of $-1 / 2$ and $-3 / 2(\mathbf{a}, \mathbf{b})^{56}$. Nodal trefoil knot and pigtail braid knot OVs (c, d) and corresponding phase distributions $(\mathbf{e}, \mathbf{f})^{104}$. Optical vortex knots of a threefold distorted loop (g), a trefoil knot $(\mathbf{h})$, and a pair of linked rings (i) ${ }^{103}$. $\mathbf{a}$, b From ref. ${ }^{56}$. Reprinted with permission from AAAS. c-f Reprinted by permission from Nature Physics $^{104}$, Copyright (2019). g-i Reprinted by permission from Nature Physics ${ }^{103}$, Copyright (2019)

closed loops, links and knots embedded in a light field ${ }^{102}$. As a new form of OVs, vortex knots have stimulated many experimental observation and theoretical studies on the dynamics of knotted vortices ${ }^{102,103}$. Vortex knots can also show many homologies, such as pigtail braid and Nodal trefoil knots ${ }^{104}$ as shown in Fig. $7 \mathrm{c}-\mathrm{f}$. Currently, researchers have realized the isolated manipulation and temporal control of optical vortex knots $^{104,105}$.

There are many other forms of OVs that cannot be fully covered in this paper. For instance, there are many freespace VB modes that carry OVs and OAM, such as elegant HLG beams ${ }^{106}$, Airy beams ${ }^{107}$, Pearcey beams ${ }^{108}$, and parabolic beams ${ }^{109}$. There are many morphologies of the non-beam spatial distribution of OVs with singularities fractality ${ }^{110}$. It is highly expected that many new formations of OVs will be reported and investigated in future explorations.

\section{Properties of VBs}

\section{Reflection and refraction}

The reflection of a VB generally does not satisfy the classical reflection law, i.e., the angle of incidence $\theta_{\mathrm{i}}$ does not equal the angle of reflection $\theta_{\mathrm{r}}$. Instead, the reflected light has a spatial deflection effect related to the OAM of the $\mathrm{VB}^{111}$. The difference between $\theta_{\mathrm{i}}$ and $\theta_{\mathrm{r}}$ is related to the OAM of the beam, obeying the generalized law of reflection $^{41}$

$$
\sin \left(\theta_{\mathrm{r}}\right)-\sin \left(\theta_{\mathrm{i}}\right)=\frac{\lambda}{2 \pi n} \frac{\mathrm{d} \phi}{\mathrm{d} x}
$$

where $\lambda$ and $\phi$ are the wavelength and phase of the light beam, respectively, and $n$ is the refractive index of the medium. In addition, the refraction of VBs does not satisfy Snell's law, i.e., $n_{\mathrm{t}} \sin \theta_{\mathrm{t}} \neq \mathrm{n}_{\mathrm{i}} \sin \theta_{\mathrm{i}}$. The refraction is related not only to the angles of incidence and refraction $\left(\theta_{\mathrm{i}}\right.$ and 
$\left.\theta_{\mathrm{t}}\right)$ and the refractive indices but also to the OAM, obeying the generalized law of refraction ${ }^{41}$

$$
\sin \left(\theta_{\mathrm{t}}\right) n_{\mathrm{t}}-\sin \left(\theta_{\mathrm{i}}\right) n_{\mathrm{i}}=\frac{\lambda}{2 \pi} \frac{\mathrm{d} \phi}{\mathrm{d} x}
$$

\section{Interference}

For conventional laser beams, the equal-inclination interference pattern is equispaced fringes, and the equalthickness interference pattern is Newton's rings. However, for a VB, the pattern of equal-inclination interference with a plane wave is not equispaced fringes but fringes with bifurcation at the singularity of the vortex, and the morphology of the bifurcation is related to the OAM of the beam $^{66}$. The equal-thickness interference pattern of a VB with a plane wave is not Newton's rings but spiral stripes extending outward from the vortex singularity, the number of which is related to the $\mathrm{OAM}^{112}$. The selfinterference pattern can also show some bifurcation fringes $^{112}$. These special interference fringes can be used in detection and measurement methods of vortices.

\section{Diffraction}

VBs have unique diffraction properties, the aperture diffraction patterns of which are coupled with the actual OAM. Since Hickmann et al. ${ }^{40}$ unveiled in 2010 the exotic lattice pattern in triangular-aperture far-field diffraction of VBs, it has been used as an effective method for OAM detection and measurement of femtosecond vortices ${ }^{113}$, non-integer charge vortices ${ }^{114}$, and elliptical $\mathrm{VBs}^{115}$. Many other unique far-field diffraction patterns were investigated through a slit ${ }^{116}$, a square aperture ${ }^{117}$, a diamond-shaped aperture ${ }^{118}$, a circular aperture ${ }^{119}$, an off-axis circular aperture ${ }^{120}$, an isosceles right triangular aperture $^{121}$, a sectorial screen ${ }^{122}$, and so on. The Fresnel diffraction of VBs was also studied ${ }^{123}$. Some special VBs, such as vector $\mathrm{VBs}^{124}$ and $\mathrm{SU}(2) \mathrm{VBs}^{97}$, can even bring about special lattice structures in diffraction patterns. These special diffraction patterns can be used in vortex detection and measurement methods.

\section{Polarization}

The polarization states of conventional beams can be represented on the Poincaré sphere. VBs can have complex transverse structures involving polarization vortices. Upon combining structured polarization with VBs, the vector VBs can demonstrate more amazing properties and more extensive applications ${ }^{74}$. To characterize a classical family of vector VBs, Holleczek et al. proposed a classicalquantum-connection model to represent cylindrically polarized beams on the Poincaré sphere ${ }^{125}$; this model was then extended to the high-order Poincaré sphere $(\mathrm{HPS})^{126}$, which can reveal SAM-OAM conversion and more exotic vector beams, including radial and azimuthal polarization beams. In an experiment, controlled generation of HPS beams was realized ${ }^{127}$ as illustrated in Fig. 8f. As an improved formation of the HPS, the hybridorder Poincaré sphere was theoretically proposed ${ }^{128}$, and the corresponding experimental controlled generation methods were also presented ${ }^{129,130}$.

\section{Quantum properties}

Twisted photons ${ }^{31}$ are associated with the quantum behaviour of macroscopic VBs. Akin to the conventional Heisenberg uncertainty, there is also the formation of uncertainty for twisted photons; i.e., the product of the uncertainties in the angle and the OAM is bounded by Planck's constant, $\Delta \phi \Delta L \geq \hbar / 2^{131,132}$. The general Fourier relationship between the angle and the OAM of twisted photons was also investigated ${ }^{133}$. In contrast to the polarization-entangled state with two dimensions, the OAM-entangled state can be high dimensional as $|\Psi\rangle=\sum c_{\ell}|\ell\rangle_{\mathrm{A}}|-\ell\rangle_{\mathrm{B}}{ }^{134}$. Combining the polarization and OAM of the photon, more complex SAM-OAM entangled photon pairs were realized ${ }^{47,135}$. There are many other new quantum properties related to OAM beams, such as the spin-orbit interaction ${ }^{136-138}$, the Hanbury-Brown-Twiss effect ${ }^{139}$, quantum interference $^{140,141}$, and the spin Hall effect ${ }^{142,143}$.

\section{Measurements of OVs}

As mentioned above, OVs can be measured by adopting the interference and diffraction properties of VBs. Counting the stripes and lattices in the special interferogram and diffraction patterns serves as a toolkit to measure the TC, OAM, and singularity distributions of corresponding OVs. In addition, for measuring phase vortices, one can use a spatial light modulator (SLM) to carry out phase transformations, reconstructing the target phase to detect the TC and OAM. Typical realizations include the forked diffraction grating detector ${ }^{144}$, the OAM sorter ${ }^{145}$, and spiral transformation ${ }^{146}$. For polarization vortices, the measurement should also consider the detection of the vector field. By introducing a spacevariant structure into a half-wave plate to modulate the polarization, the $\mathrm{TC}$ of the polarization singularity in vector VBs can be measured ${ }^{147}$. For measuring more properties of vector OVs, Forbes' group introduced quantum measurement methods to classical light and realized more precise measurement of properties such as the non-separability, SAM-OAM coupling, and vector factors of vector beams ${ }^{148,149}$, which is widely applicable to more structured OVs. 


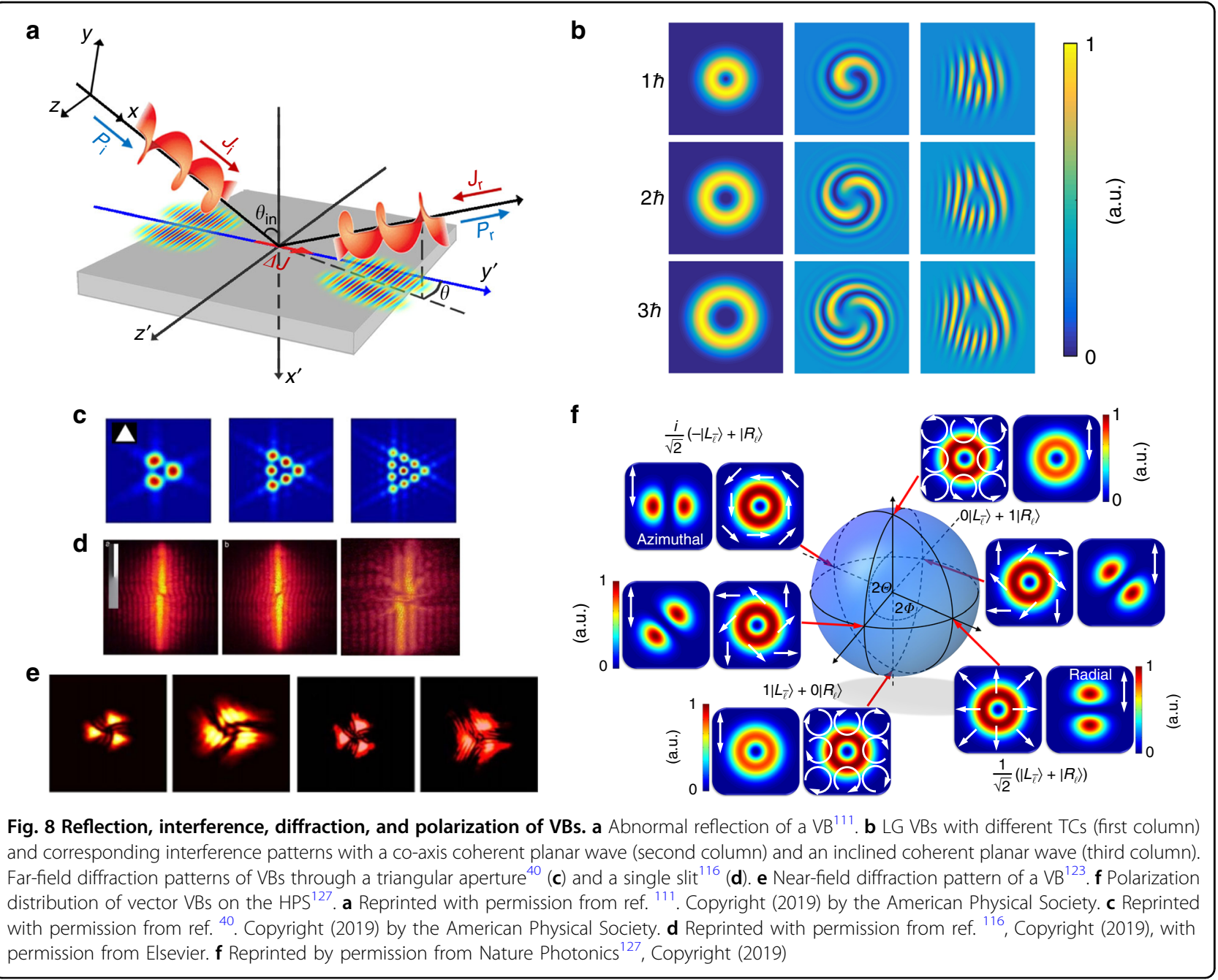

\section{Progress in vortex generation, tuning, and manipulation \\ Brief review of vortex generation}

The vortex generation methods can be divided into passive vortex generators (converting the fundamental Gaussian beams into VBs by using dynamic or geometric phase plates, metasurfaces, SLMs, etc.) and active vortex laser generators (such as free space or fibre vortex lasers and nanointegrated OAM generators $)^{61,112,150}$. There have already been some recent reviews on vortex and OAM beam generation ${ }^{61-}$ $63,112,150$. However, a review focused on vortex generation with tunable and multi-singularity properties is rare. Hereinafter, we specifically review active vortex generation with tunable properties, including wavelength-, temporal-, and OAM-tunable beams. In particular, the OAM-tunable beams include TC-tunable and multi-singularity-tunable beams.

\section{Wavelength- and OAM-tunable VBs}

OAM-tuning of VBs can be realized by gain-loss control $^{151}$, off-axis pumping ${ }^{92,152}$, or the use of a spiral phase plate (SPP) $)^{153}$, a Q-plate ${ }^{154,155}$, or an SLM ${ }^{144}$. A wavelength-tunable $\mathrm{VB}$ can be achieved by designing special liquid crystal devices ${ }^{156}$, microcavities ${ }^{157}$, or onchip gratings ${ }^{158}$ or using nonlinear frequency conver$\operatorname{sion}^{159,160}$. However, more methods to simultaneously realize wavelength and OAM tuning for novel applications, such as high-capacity optical communication using wavelength- and mode-division multiplexing, are still required.

In 2016, Zhang's group ${ }^{161}$ presented a wavelength- and OAM-tunable system by employing a tunable fibre laser with an acousto-optic fibre grating with a wavelengthtunable range of $1540-1560 \mathrm{~nm}$ and an OAM of $\pm 1 \hbar$, as shown in Fig. 9a, b. In 2017, Lyubopytov et al. ${ }^{162}$ designed a micro-electro-mechanical (MEMS) filter system 


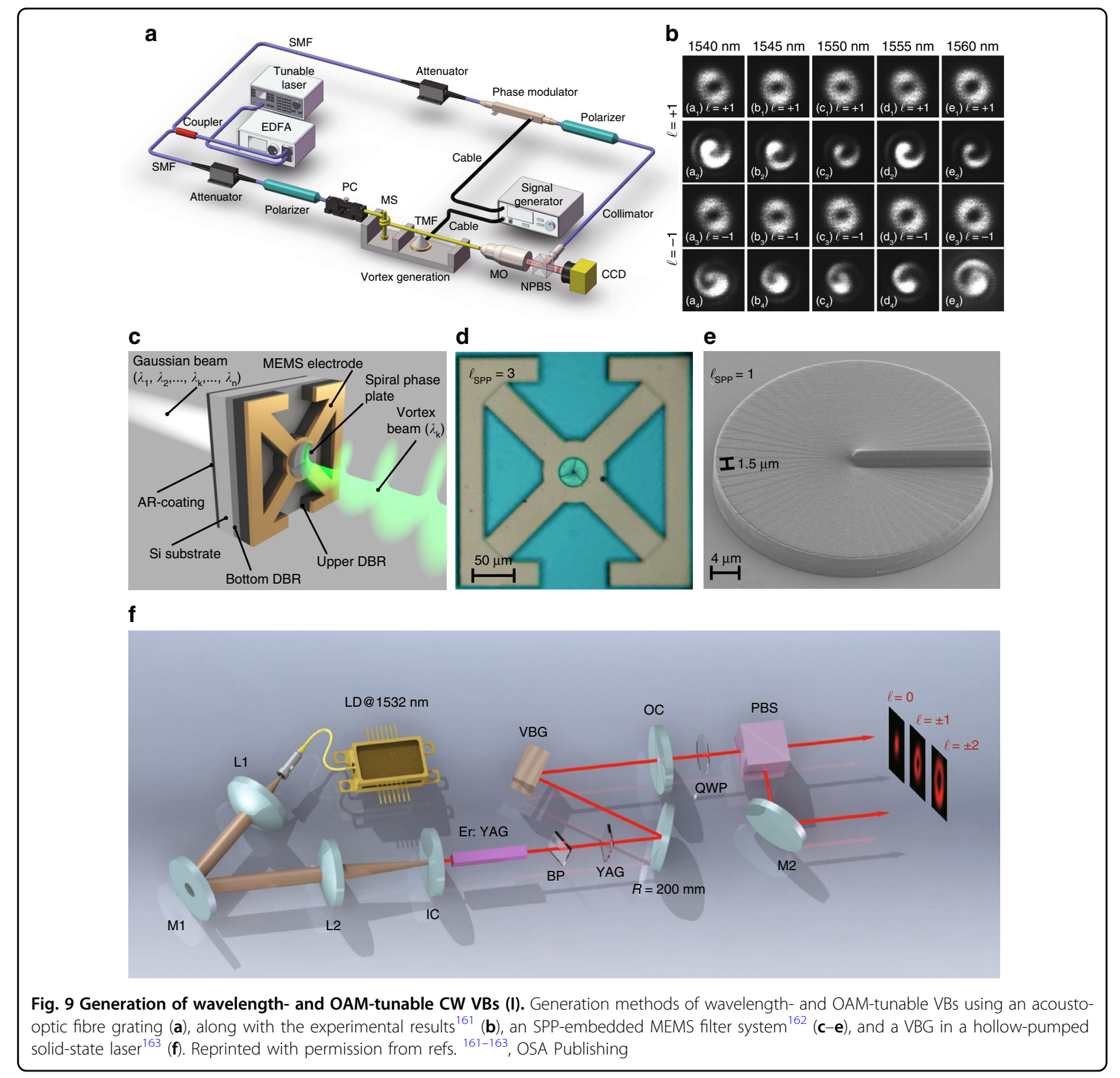

realizing vortex generation with a wavelength-tunable width of $37.5 \mathrm{~nm}$ and an OAM of $0 \sim 3 \hbar$. In the same year, Liu et al. ${ }^{163}$ reported a ring-pumped Er:YAG solid-state laser generating an 8.4- $\mathrm{nm}$ wavelength-tunable width and $0 \sim \pm 2 \hbar$ OAM-tunable VB. In 2018, Yao et al. ${ }^{164}$ invented a new optical fibre combiner for combining two polarization-controllable fundamental modes into a VB with chiral control, obtaining a 30-nm wavelength-tunable width and $0 \sim \pm 1 \hbar$ OAM. Our group ${ }^{165}$ proposed solid-state vortex generation utilizing a dual-off-axis pumped ultra-wide-band Yb:CALGO laser, reaching a wavelength-tunable width of over $10 \mathrm{~nm}$ and an OAM range of $0 \sim \pm 15 \hbar$, as depicted in Fig. 10a. This system was adapted to generate tunable dual-wavelength $\mathrm{VBs}^{166}$. Recently, Wang et al. ${ }^{167}$ improved the output efficiency and reduced the threshold of a similar system by using a Z-cavity and a birefringent plate in the cavity design, and a 14.5-nm wavelength-tunable width and a $0 \sim \pm 14 \hbar$ OAM range were achieved. Wang's group ${ }^{168}$ designed and implemented a fibre-space coupling vortex laser system, where a wavelength-tunable range of 1530-1565 $\mathrm{nm}$ and an OAM of $0 \sim \pm 10 \hbar$ were achieved. 


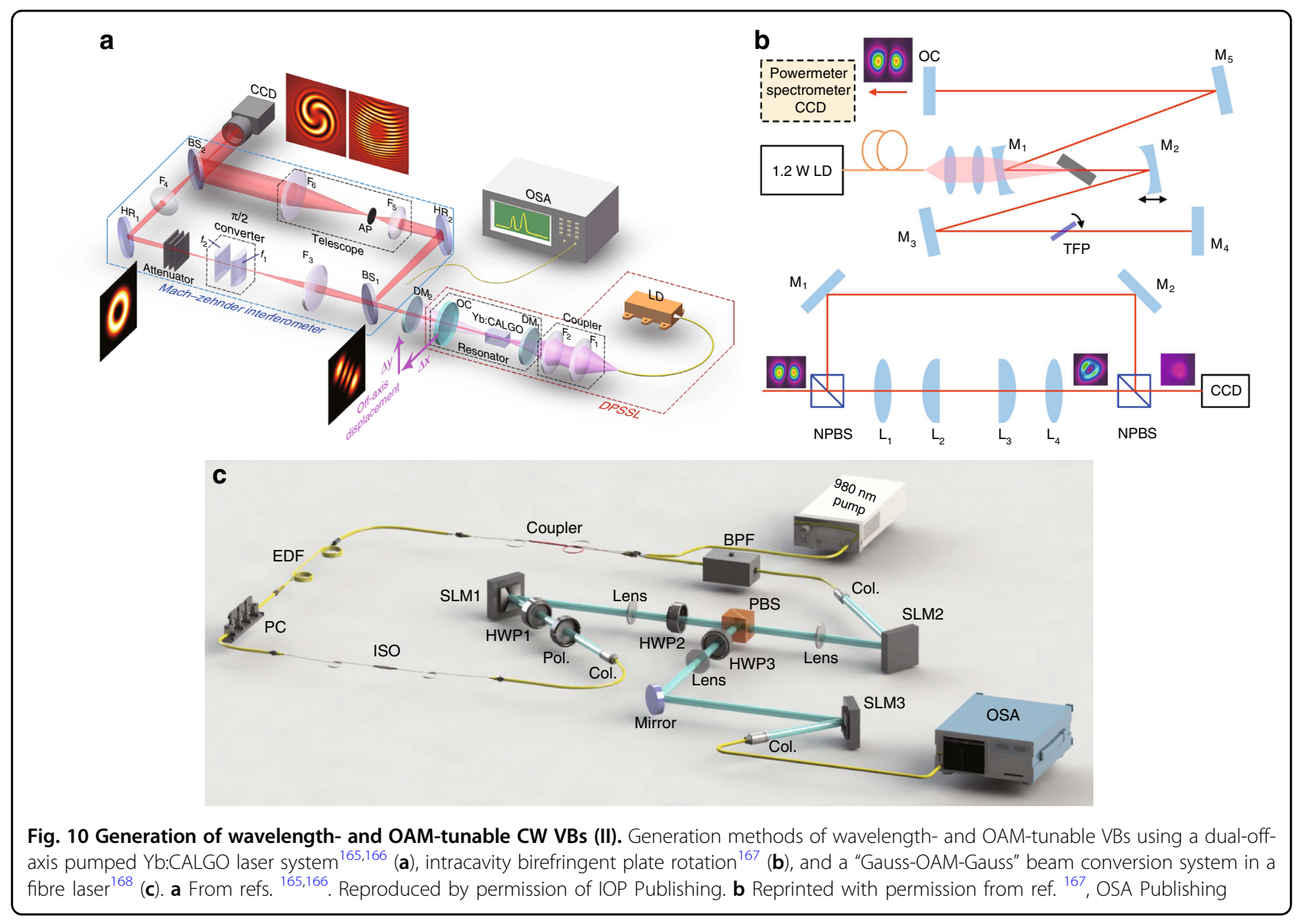

In addition to the abovementioned wavelength- and OAM-tunable OVs from lasers, there are vortex generators that are known to be intrinsically broadband, which can also be used to obtain wavelength- and OAMtunable OVs. For instance, vortex generation from anisotropic solid crystals, both uniaxial and biaxial, can be related to complex SAM-OAM coupling and gain competition effects, leading to the tunability of vector $\mathrm{OVs}^{72,73,169}$. Similar tunable OVs can be generated in chiral liquid crystals in the regime of circular Bragg reflection ${ }^{170,171}$. Taking advantage of space-variant anisotropic liquid crystals that can be electrically controlled, wavelength- and OAM-tunable OVs can be generated within a wide spectral tunable range ${ }^{156,172}$. Overall, a wider tunable range controlled by a more convenient $\mathrm{OV}$ generation method is still required in the current explorations.

\section{Pulsed VBs}

High-peak-power pulsed VBs with different levels of duration have great potential for use in advanced applications, such as optical machining ${ }^{173,174}$, nonlinear optics $^{25,26,48,49}$, strong-field physics ${ }^{175}$, and optical tweezers ${ }^{176}$. Hereinafter, we review the recent compact and effective pulsed VB sources.

\section{Nanosecond-level VBs}

The generation of nanosecond-level VBs has always been combined with Q-switched lasers. An earlier method involved selecting the LG modes using an intracavity aperture in a Q-switched solid-state laser ${ }^{177}$, with which obtaining high mode purity is difficult. In 2013, Kim et al. ${ }^{178}$ used an etalon and a Brewster plate in an acoustic-optic Q-switched laser and generated highquality chirality-controlled LG beams with an $\sim 250 \mu \mathrm{J}$ pulse energy and an $\sim 33 \mathrm{~ns}$ duration. In 2016, Zhao et al. ${ }^{179}$ controlled the pump position in an Er:YAG acoustic-optic Q-switched laser, generating a nearly 1-mJ and 50-ns pulsed VB. In 2017, Chen's group ${ }^{180}$ designed a nanosecond vortex laser system employing a compact $\mathrm{Nd}$ : $\mathrm{YVO}_{4} / \mathrm{Cr}^{4+}$ :YAG passively Q-switched laser with an external AMC. In 2018, He et al. ${ }^{181}$ presented a Cr,Nd: YAG self-Q-switched microchip laser to directly generate low-threshold nanosecond high-repetition-rate vortex pulses without an AMC, where the chirality was 
controlled by a tilted output mirror. Our group ${ }^{182}$ recently reported a pulsed vortex output directly from a room-temperature diode-pumped Er,Yb:glass microchip laser with an extremely compact structure.

\section{Picosecond-level VBs}

Picosecond-level VBs have always been realized in a mode-locking laser using a narrow-band gain medium. In 2011, Koyama et al. ${ }^{183}$ realized a VB in a stressed Ybdoped fibre amplifier seeded by a picosecond mode-locked $\mathrm{Nd}: \mathrm{YVO}_{4}$ laser with a pulse width of $8.2 \mathrm{ps}$ and a peak power of $34.2 \mathrm{~kW}$. However, the master oscillator poweramplifier structure limited the compactness. The discovery of a self-mode-locking effect in neodymium-doped crystals provided an alternative way to generate picosecond pulses with a quite compact structure ${ }^{184}$. In 2009, Liang et al. ${ }^{185}$ reported an OV with a pulse width of $20-25$ ps and a repetition rate of $3.5 \mathrm{GHz}$ using off-axis-pumped selfmode-locked Nd:GdVO 4 lasers with an AMC. In 2013, the same group ${ }^{186}$ improved this system via cavity control and realized the self-mode-locked $\mathrm{SU}(2)$ vortex GM with pulse widths of $22.2 \mathrm{ps}$ and $21.1 \mathrm{ps}$ for $\Omega=1 / 4$ and $\Omega=1 / 3$, respectively $^{92}$. In 2017, Tung et al. ${ }^{187}$ reported the direct generation of picosecond large-OAM SU(2) vortex pulses in self-mode-locking $\mathrm{Nd}: \mathrm{YVO}_{4}$ lasers without the help of an $\mathrm{AMC}$, which largely enhanced the compactness. In 2018, Huang et al. ${ }^{188}$ reported an 8.5 ps pulsed VB generated from a mode-locked fibre laser, where the polarization could be controlled at arbitrary states on the HPS.

\section{Femtosecond-level VBs}

In contrast to picosecond pulses, femtosecond pulse generation always requires more extreme operating conditions, such as a tightly focused pumping spot, a wide emission band, and a high nonlinear coefficient of the gain medium. Utilizing the external modulation method, flexible temporal shaping of femtosecond VBs was recently realized $^{189}$. Considering the improvements in compactness and cost, the self-mode-locking laser oscillator scheme is still desirable. In 2013, Chen's group ${ }^{190}$ reported a self-mode-locked monolithic Yb:KGW laser with a duration of $850 \mathrm{fs}$ and a repetition rate of $22.4 \mathrm{GHz}$. In 2016, they ${ }^{191}$ improved the system to directly generate a sub-picosecond VB carrying OAM by selective pumping. In 2018, Zhang et al. ${ }^{192}$ proposed an all-fibre mode-locked femtosecond $\mathrm{LG}_{0, \pm 1}$ vortex laser with a pulse width of 398 fs. In the same year, Wang et al. ${ }^{193}$ realized direct emission of an ultrafast $L_{0, \pm 1} \mathrm{VB}$ via a $\mathrm{z}$-type cavity design in an SESAM mode-locking Yb:QX laser with a pulse width of $360 \mathrm{fs}$, as shown in Fig. 11c. These structures have recently been improved by using a Yb:KYW oscillator with a defect-spot mirror, obtaining a 298-fs $\mathrm{VB}^{194}$. Direct generation of sub-100-fs VBs may be a future target.

\section{Complex OAM manipulation}

In addition to TC-tunable VBs, beams with multiple singularities can induce exotic tunable OAM. The multisingularity optical field with a vortex array is also known as a vortex lattice or a vortex crystal $^{5,29,73}$. Strong requirements of multi-singularity beams have been put forward because of the boom of special applications such as multiple particle manipulation ${ }^{82,195}$, 3D displays ${ }^{196}$, and optical modulation and communication ${ }^{197}$.

A singularity splitting phenomenon was found in an $\mathrm{AMC}$ when the phase matching condition in the AMC was not satisfied $^{198,199}$. A large number of matrix optics theories were put forward ${ }^{200,201}$, deriving the HLG mode to describe the controllable generation of a vortex array in the AMC system $^{76,77}$. Similar to the HLG mode, the HIG mode is also a multi-singularity $\mathrm{VB}$, which can be generated in special cavities with selective pumping ${ }^{80}$ and an $\mathrm{SLM}^{81}$. Recently, our group proposed a method of tuning the periodic orbits of an SU(2) GM in a degenerate cavity and further tuning the multi-singularity OAM of $\mathrm{SU}(2) \mathrm{VBs}^{202-204}$, as shown in Fig. 12b. In addition to HIG, HLG and SU(2) VBs, many other multi-singularity VBs with special mathematical formulations were generated with different control methods, such as trochoidal $\mathrm{VBs}^{95}$, transverse-mode-locking vortex lattices ${ }^{202,203}$, and polygonal $\mathrm{VBs}^{96}$.

In addition to the above multi-singularity modes, people are pursuing more freely tailored methods for arbitrary singularity distributions. SLM modulation combined with a laser source for on-demand modes has been favoured ${ }^{205}$. Recently, increasing numbers of tailored singularity distributions have been designed and realized via SLMs, such as rectangular and circular multi-singularity arrays ${ }^{206,207}$ and arbitrary curvilinear arrays ${ }^{208}$, and quadrantseparable singularity control ${ }^{209}$, as presented in Fig. 13.

There are still many novel methods of tuning the multi-singularity OAM in more types of exotic OVs. For instance, various $\mathrm{OV}$ arrays can be generated by coherent combining technology with digital control ${ }^{210}$. Infinite scalar and vector OV arrays can be realized in fractional OAM VBs ${ }^{211,212}$. On-demand multi-singularity VBs can be generated based on the appropriate combination of optical scattering and discrete rotational symmetries of optical isotropic masks ${ }^{213}$ and can be electrically and optically controlled via anisotropic masks ${ }^{214,215}$.

Despite the numerous multi-singularity manipulation methods, the realization of universal and versatile tunability will be the everlasting target in the future.

\section{Advanced applications of tunable VBs Optical tweezers}

Optical tweezers that trap particles using an optical force were proposed by Ashkin ${ }^{216}$, who won the Nobel Prize in 2018. Benefitting from the study of OAM 

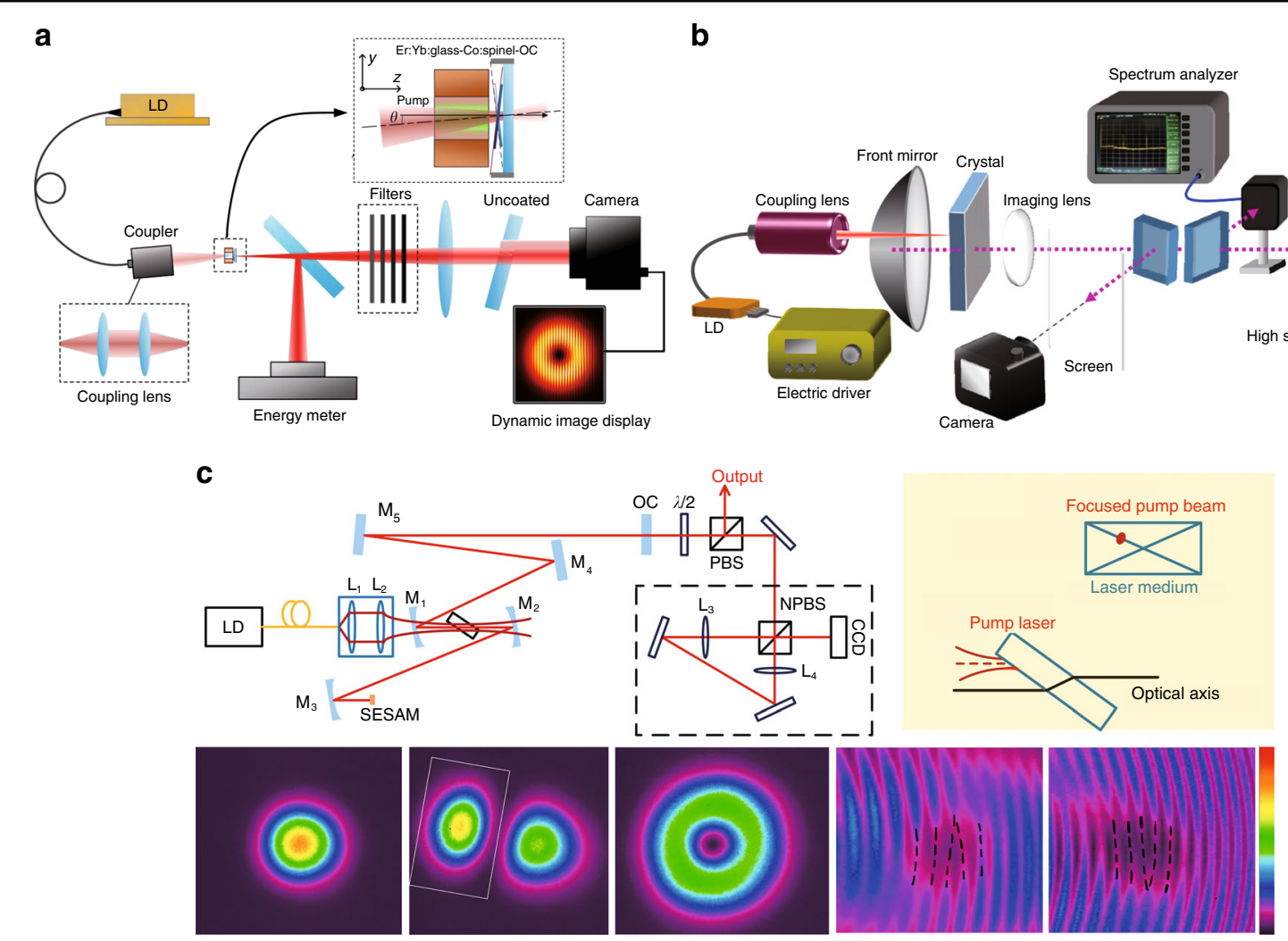

Fig. 11 Generation of wavelength- and OAM-tunable pulsed VBs. Generation methods of a room-temperature diode-pumped Er,Yb:glass microchip nanosecond laser ${ }^{182}$ (a), picosecond-level VBs in a self-mode-locked Nd:YVO laser $^{187}$ (b), and femtosecond VBs in an SESAM mode-locking laser $^{193}$ (c). a $\odot(2019)$ IEEE. Reprinted, with permission, from ref. ${ }^{182}$. b Reprinted with permission from ref. ${ }^{187}$, OSA Publishing. c From ref. ${ }^{193}$. Reproduced by permission of IOP Publishing

interactions with matter, OVs were first used in 1995 in optical tweezers and extended to the optical spanner ${ }^{19}$, where particles can be trapped and driven to move around the singularity. Then, the transformation from optical OAM to mechanical AM was widely studied ${ }^{32-34}$.

With the improvement of vortex tunability, newgeneration tweezers with OVs have shown distinct advantages $^{34,217}$. As demonstrated in Fig. 14, the novel vortex tweezers can conveniently manipulate not only the spatial positions of particles but also the multiple degrees of freedom of particles, largely extending the automated guiding, assembly, and sorting technology $y^{217,218}$. With the control of multi-singularity VBs, many new techniques were designed and applied to trap multiple particles $^{82,217,218}$, including the fractional optical VB for optical tweezers ${ }^{219}$. With femtosecond VBs, the tweezers carrying special nonlinear properties can be used to manipulate optical Rayleigh particles ${ }^{220}$. Furthermore, with femtosecond vector VBs, nonlinearity-induced multiplexed optical trapping and manipulation was designed ${ }^{221}$, where the number of traps and their orientations could be flexibly controlled. In addition to dielectric particles, metal particles can also be manipulated by novel plasmonic vortex tweezers ${ }^{222}$, where the vortex field of surface plasmon polaritons can be generated by focusing vector VBs onto a metal film. Plasmonic vortex tweezers as depicted in Fig. 15d, e were shown to be superior in manipulating metal particles with large flexibility ${ }^{223}$.

\section{Optical communication}

In addition to the polarization, amplitude, pulse shape, and wavelength of light, the OAM can be used as an alternative degree of freedom for multiplexing modulation, enlarging the capacity of optical communication ${ }^{39}$, which is also referred to as mode/spatial-division multiplexing $(\mathrm{MDM} / \mathrm{SDM})^{224}$. Optical communication by OAM multiplexing has enabled breaking the Tbit level ${ }^{43,44}$, much beyond the conventional scheme, thus greatly broadening the application scope ${ }^{225,226}$. With the study of VB propagation in the atmosphere, free-space communication using vortices was gradually improved $^{227-229}$. Furthermore, a sidelobe-modulated OV method was proposed for free-space communication with a significant increase in the data transmission capacity ${ }^{230}$. 


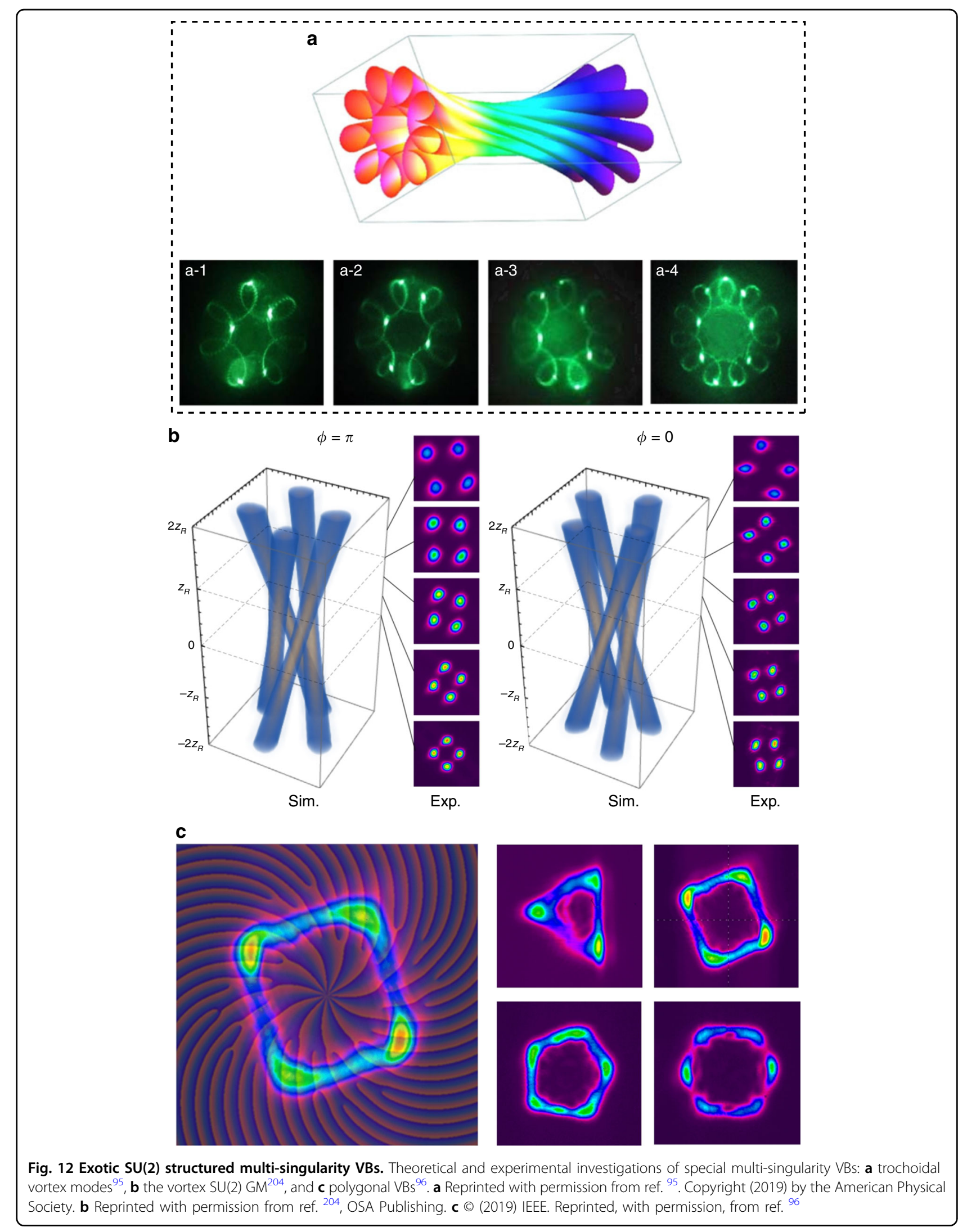



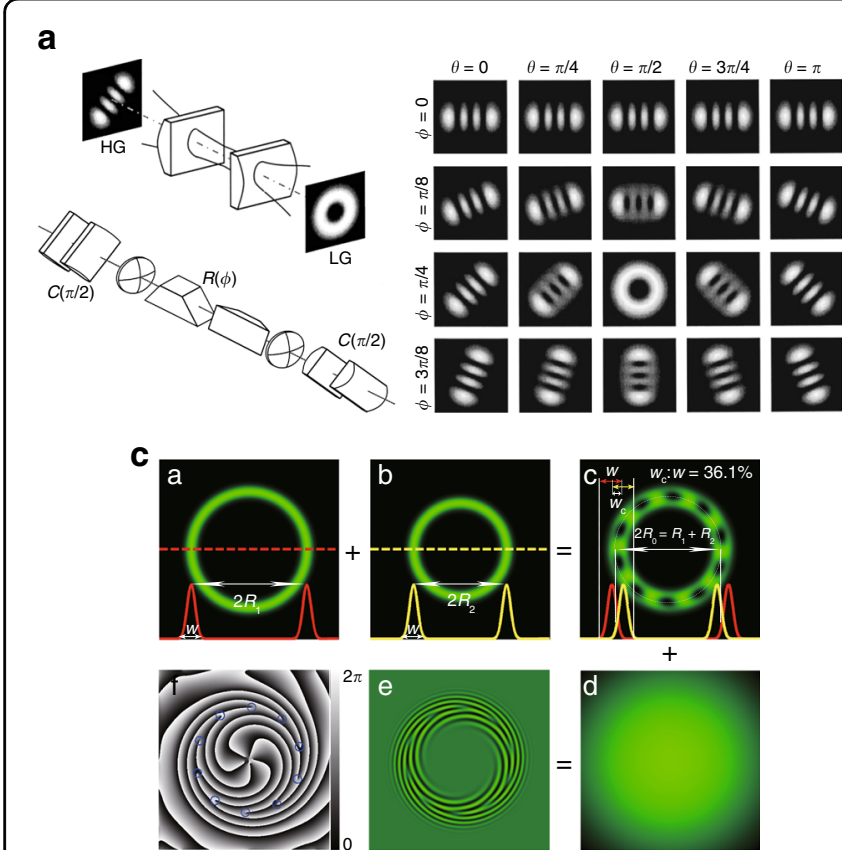

Fig. 13 Generation of multi-singularity VBs. a Exploring the singularity splitting phenomenon in AMC systems ${ }^{200}$. $\mathbf{b}$ Multiple singularity formation in fractional OAM VBs ${ }^{211}$. Tailoring multi-singularity beams with $\mathbf{c}$ a circular vortex array ${ }^{207}$ and $\mathbf{d}$ an optical vortex array along an arbitrary curvilinear arrangement ${ }^{208}$. a Reprinted with permission from ref. ${ }^{200}$, Copyright (2019), with permission from Elsevier. b Reprinted with permission from ref. ${ }^{211}$, OSA Publishing. c Reprinted with permission from ref. ${ }^{207}$. Copyright (2019) by John Wiley and Sons. d Reprinted with permission from ref. ${ }^{208}$, OSA Publishing
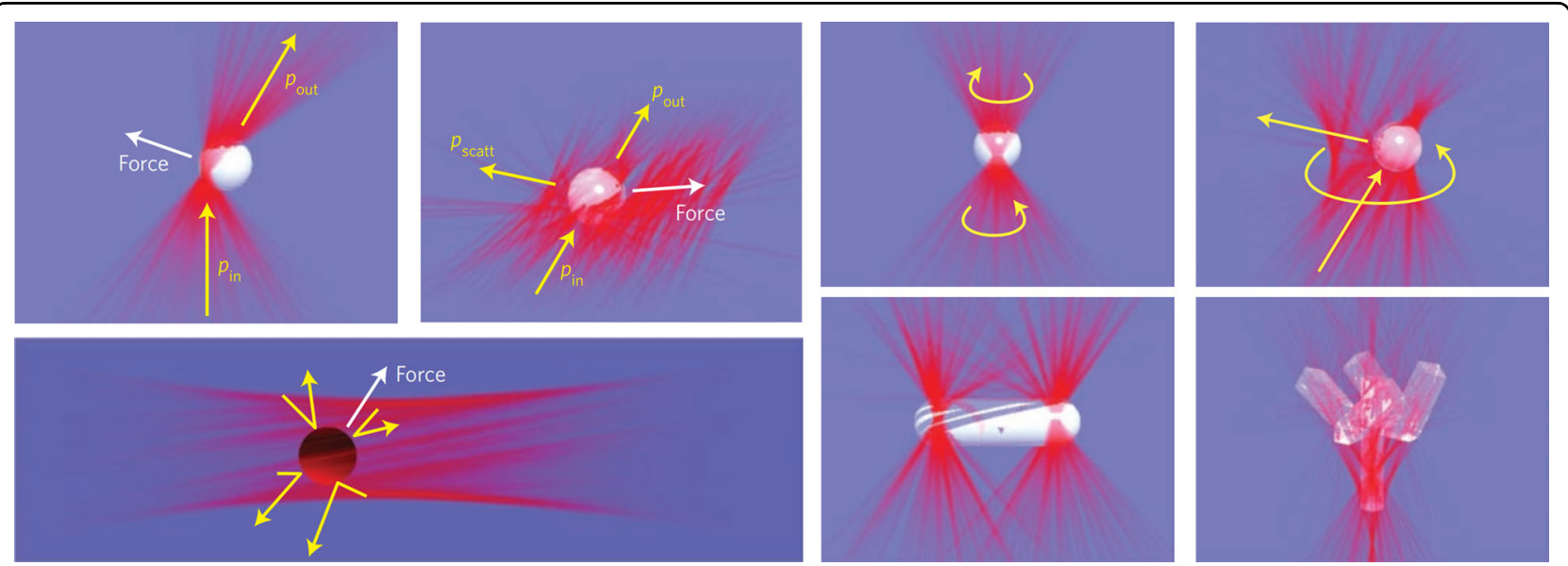

Fig. 14 Optical tweezers with VBs can manipulate not only the positions of particles but also their motion, such as precession, nutation, spin, and more complicated orbital motion ${ }^{217}$. Reprinted by permission from Springer Nature: Nature Photonics ${ }^{217}$, Copyright (2019)

With the development of multi-singularity-tunable VBs, the capacity and speed of communication can be further improved $^{231}$. A variety of special fibres for OAM mode transmission were designed to enable fibre-based vortex communication technology 232,233 . Recently, a new OAM multiplexing technology using Dammann vortex gratings in fibre-free-space coupled systems realized massive
OAM state parallel detection ${ }^{234}$, offering an opportunity to raise the communication capacity to the Pbit level. OAM-multiplexing-based communication was also demonstrated under many extreme circumstances, such as underwater communication ${ }^{235}$ illustrated in Fig. 16d, high-dimensional quantum communication ${ }^{236}$, and longdistance fibre communication ${ }^{237}$. 

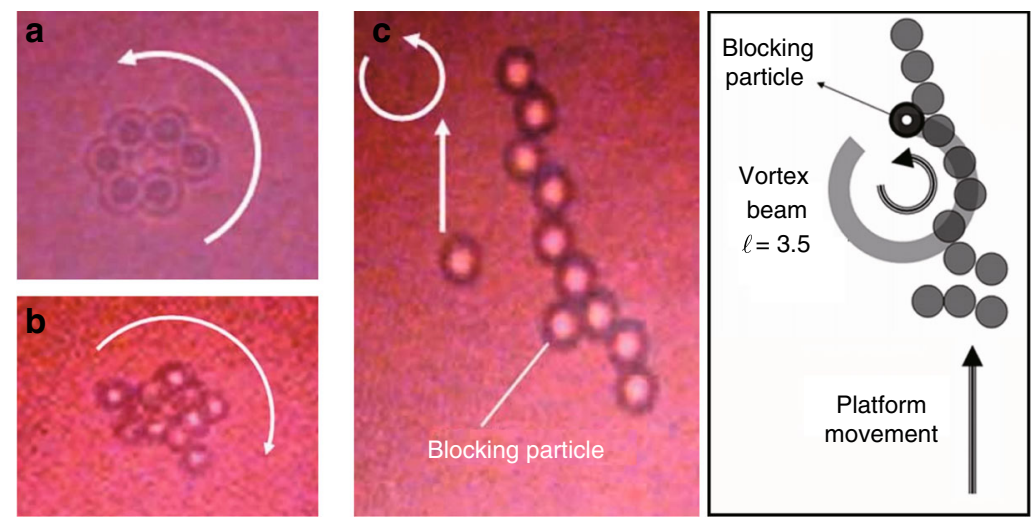

d

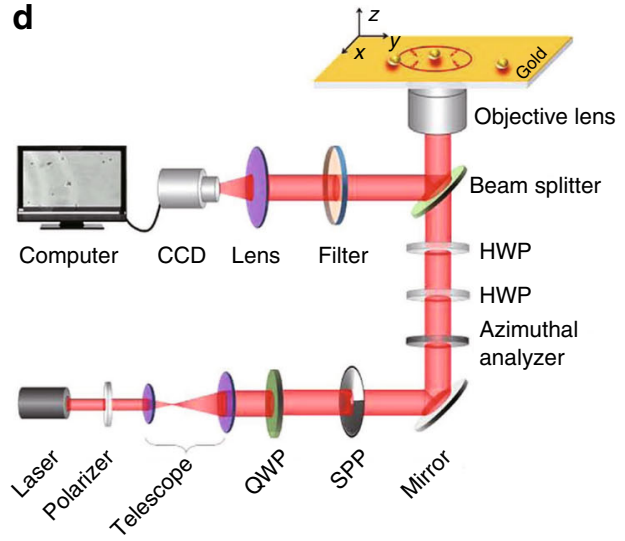

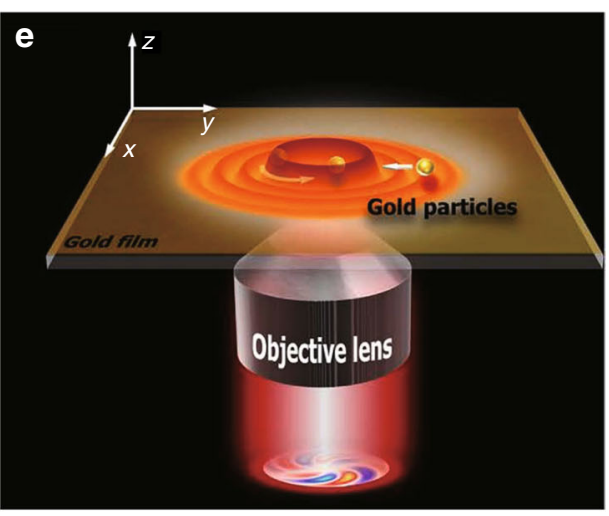

Fig. 15 Particle manipulation using OVs. a, b Manipulating the rotation of multiple particles and $\mathbf{c}$ aligning and transporting particles with fractional $\mathrm{VBs}^{219}$. d Setup and e schematic of plasmonic vortex tweezers for manipulating metal particles ${ }^{223}$. a-c Reprinted with permission from ref. ${ }^{219}$, OSA Publishing

\section{Quantum entanglement}

With the recent mature quantum descriptions of twisted photons ${ }^{31}$, OAM entanglement has engendered plenty of applications ${ }^{134}$. For instance, high-dimensional quantum key distribution (QKD) protocols can be designed based on mutually unbiased bases related to OAM photons $^{238}$, which motivated high-dimensional quantum cryptography for high-security communication ${ }^{239}$. The quantum memory technology for OAM photonic qubits was recently proposed to provide an essential capability for future networks ${ }^{240}$. Because of the inherent infinite dimension of OAM, the OAM of photons has been successfully used to realize quantum storage in various systems, such as atomic ensembles ${ }^{241}$ and rare-earth-iondoped crystals $^{242}$, benefiting high-capacity communication. High-dimensional OAM entanglement was also successfully used in high-efficiency digital spiral imaging $^{243}$. Employing the Hong-Ou-Mandel interference of OAM photons, quantum cloning technology for making copies of unknown quantum states was presented ${ }^{244}$. With the development of vector VB manipulation, SAM and OAM were combined for quantum communication to further scale the capacity and speed ${ }^{245}$. Quantum teleportation using OAM can largely improve the technical control of scalable and complicated quantum systems ${ }^{246}$. To date, the entangled photon system with the highest number of qubits (18 qubits with six entangled photons) with OAM as one degree of freedom has been produced $^{247}$. Very recently, as a remarkable breakthrough, quantum entanglement between the SAM and OAM states was realized in a metamaterial ${ }^{47}$.

In addition to scalar phase OVs, vector polarization OVs also have fruitful quantum properties. The non-separable states between the polarization and space share common properties with the entangled state of photons, which is also called the classical entanglement state ${ }^{71,248}$. The quantum tomography, Bell parameter, concurrence count, and linear entropy can be realized in vector OVs akin to corresponding quantum measurements ${ }^{148,149,248}$. Taking advantage of the high-dimensional properties of the nonseparable states, quantum walks can be implemented by vector OV modes of light, enlarging the scalable range ${ }^{249}$. Entanglement beating generated in vector VBs can be used to control spin-orbit coupling in free space ${ }^{135}$. High- 


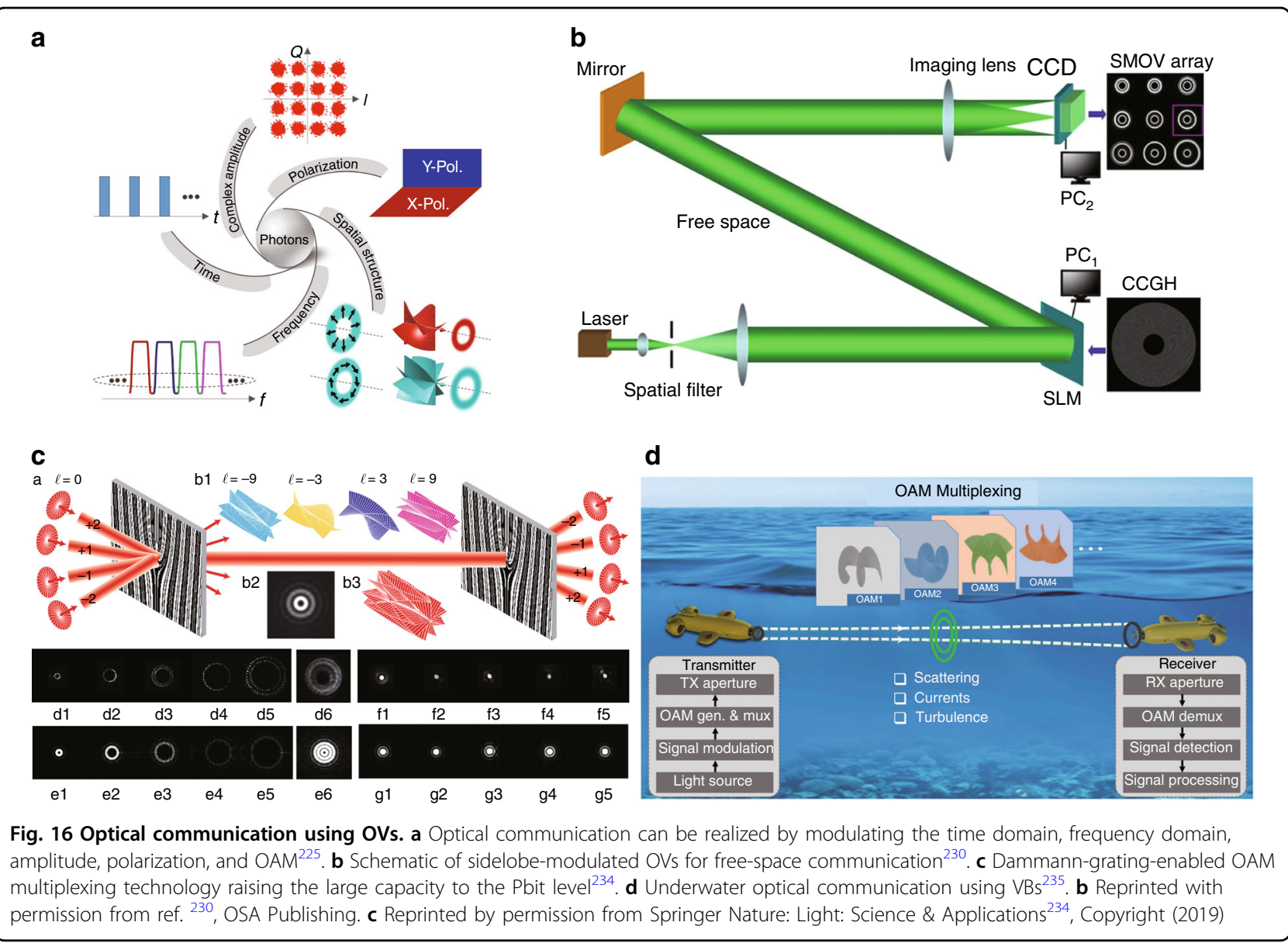

dimensional entanglement has also been utilized in coding quantum channels to improve high-capacity optical communication $^{250}$, as illustrated in Fig. 17d, e.

\section{Nonlinear optics}

With the development of high-power and large-energy $\mathrm{VBs}^{92,180,251}$, the peak power can exceed the threshold of various nonlinear effects, providing conditions to explore novel nonlinear conversion phenomena related to $\mathrm{OAM}^{48,49,251}$. Conventionally, the development of nonlinear optics was based mainly on the scattering that obeys momentum conservation (Rayleigh scattering, Brillouin scattering, Raman scattering, etc.), and the corresponding development of nonlinear frequency transformation effects (frequency doubling, frequency summing, four-wave mixing, etc.) has benefited a myriad of applications. In the new century, new transverse nonlinear transformation effects have been developed based on AM conservation, such as TC variation during the processes of frequency doubling ${ }^{25,26}$, summing and mixing ${ }^{252,253}$, tunable OAM highharmonic transform ${ }^{48,49}$, and OAM strong-field physics ${ }^{175}$. Recently, these OAM harmonic generations have been widely applied in nanomaterials for the control of nonlinear phases $^{254}$, the Pancharatnam-Berry phase ${ }^{255}$ and beam shaping ${ }^{256}$. In addition, there are many novel physical phenomena coupled with nonlinear OAM-frequency conversion, such as the rotational Doppler effect ${ }^{257}$ and rotational nonlinear absorption ${ }^{258}$.

\section{Nanotechnology}

Due to the rapid development of nanofabrication and increasing demands for nanotechnology applications, nanointegrated on-chip vortex generators have emerged for emitting VBs at the nanoscale, such as integrated silicon-chip-based VB emitters ${ }^{259}$, vortex vertical-cavity surface-emitting lasers (VCSELs) ${ }^{260}$, angular gratings ${ }^{42}$, micro-nano-OAM laser emitters ${ }^{261}$, and various metasurface designs ${ }^{262}$. Taking advantage of nanoscale VBs, many novel phenomena related to OAM in nanophotonic materials have been demonstrated, such as non-dispersive vortices $^{263}$ and SAM-to-OAM conversion effects ${ }^{46,47}$. Combined with new nanomaterials, many vortex-emitting materials and devices with unique functions have been invented, such as vector vortex on-chip generators ${ }^{264}$ and parallel OAM processors ${ }^{265}$. Combining quantum technology and nanotechnology, a photonic chip capable of 


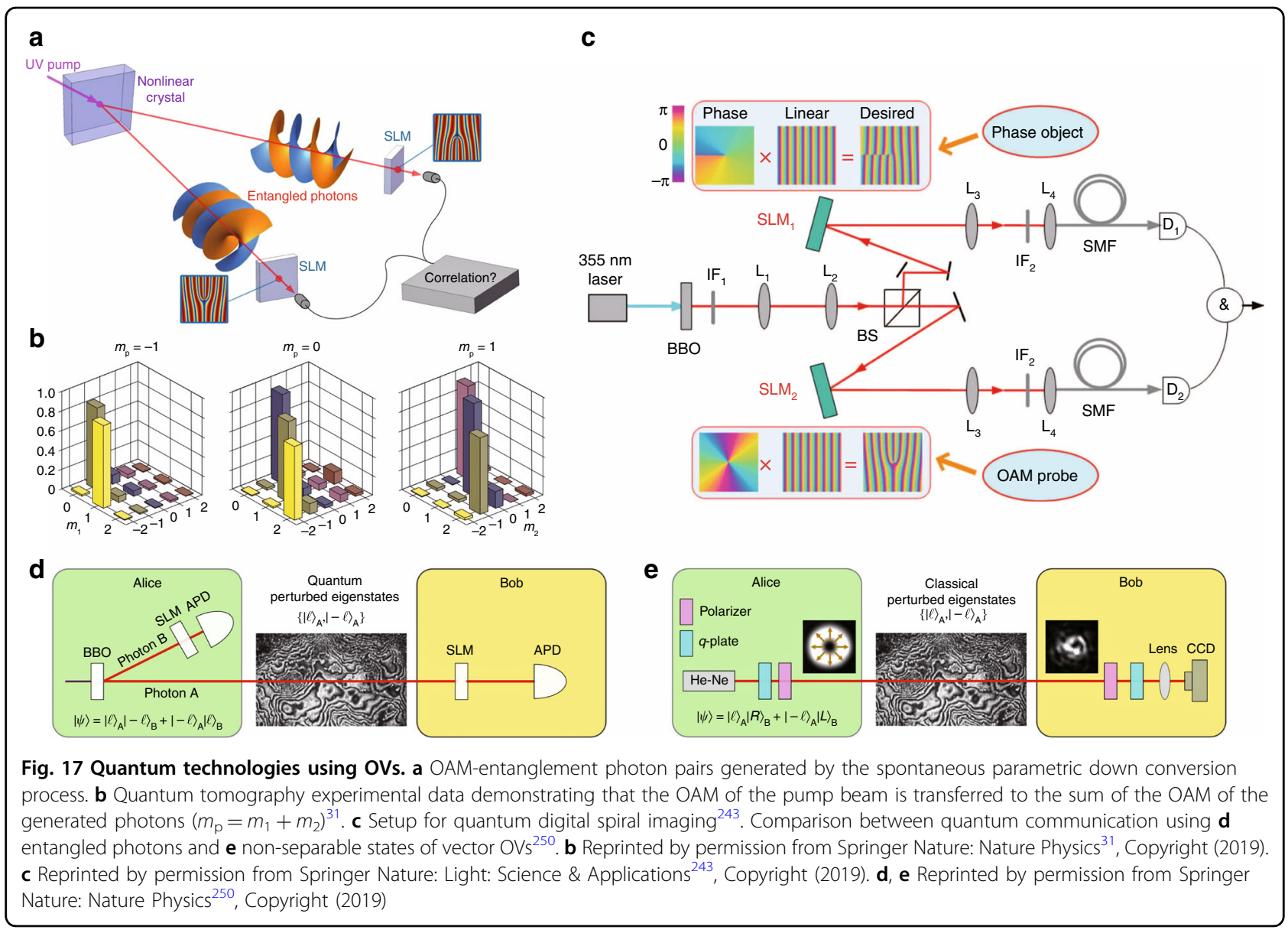

purifying the OAM quantum states was recently produced, which possesses great potential to develop on-chip quantum calculation ${ }^{266}$.

\section{Optical machining}

Due to the nature of high-order modes, VBs show weaker capability in conventional machining processes, such as laser cutting and laser punching, than the fundamental Gaussian beam. However, in some special applications, vortex light has distinct advantages. When a metal surface is processed by different vector VBs, various intriguing new patterns can be selectively displayed under light illumination ${ }^{267,268}$. Moreover, the surface can exhibit different patterns when the illuminated light has different incidence angles ${ }^{269}$. In addition to the angular sensitivity, a polarization-sensitive surface was fabricated based on a similar technique using vortex processing, i.e., different patterns were exhibited when the surface was illuminated by light with different polarizations ${ }^{268}$. Utilizing nanophotonics technology, nanoscale VBs were used in nanostructure fabrication. For instance, the chiral nanoneedle structure can be easily fabricated by a perpendicular VB through the transfer of the consequential torque from OAM light to the object ${ }^{173,269,270}$. Similar methods can produce some other nanostructures, such as helical surface reliefs ${ }^{271}$ and monocrystalline silicon needles ${ }^{272}$. Recently, high-power ultrashort OAM-tunable VBs were combined with femtosecond laser direct writing technology to process more special structures, such as multiwaveguide $^{266}$ and micro-pipe structures ${ }^{174}$.

\section{Microscopy and imaging}

The unique spiral phase of VBs can be used in phasecontrast microscopy, demonstrating high-resolution micro-imaging ${ }^{37}$. Applying OAM analysis in the imaging method, the novel digital spiral imaging technique was proposed to improve the resolution ${ }^{273}$. Currently, imaging using OAM has already realized super-diffraction-limit resolution $^{38}$. In recent years, a growing number of novel microscopy and imaging technologies using VBs have emerged, reaching increasingly higher resolution. For instance, plasmonic structured illumination microscopy using standing surface plasmon waves induced by OVs was proposed, realizing high-resolution wide-field imaging $^{274}$. This microscopy was further improved by using perfect VBs (VBs with a controllable ring radius) to 


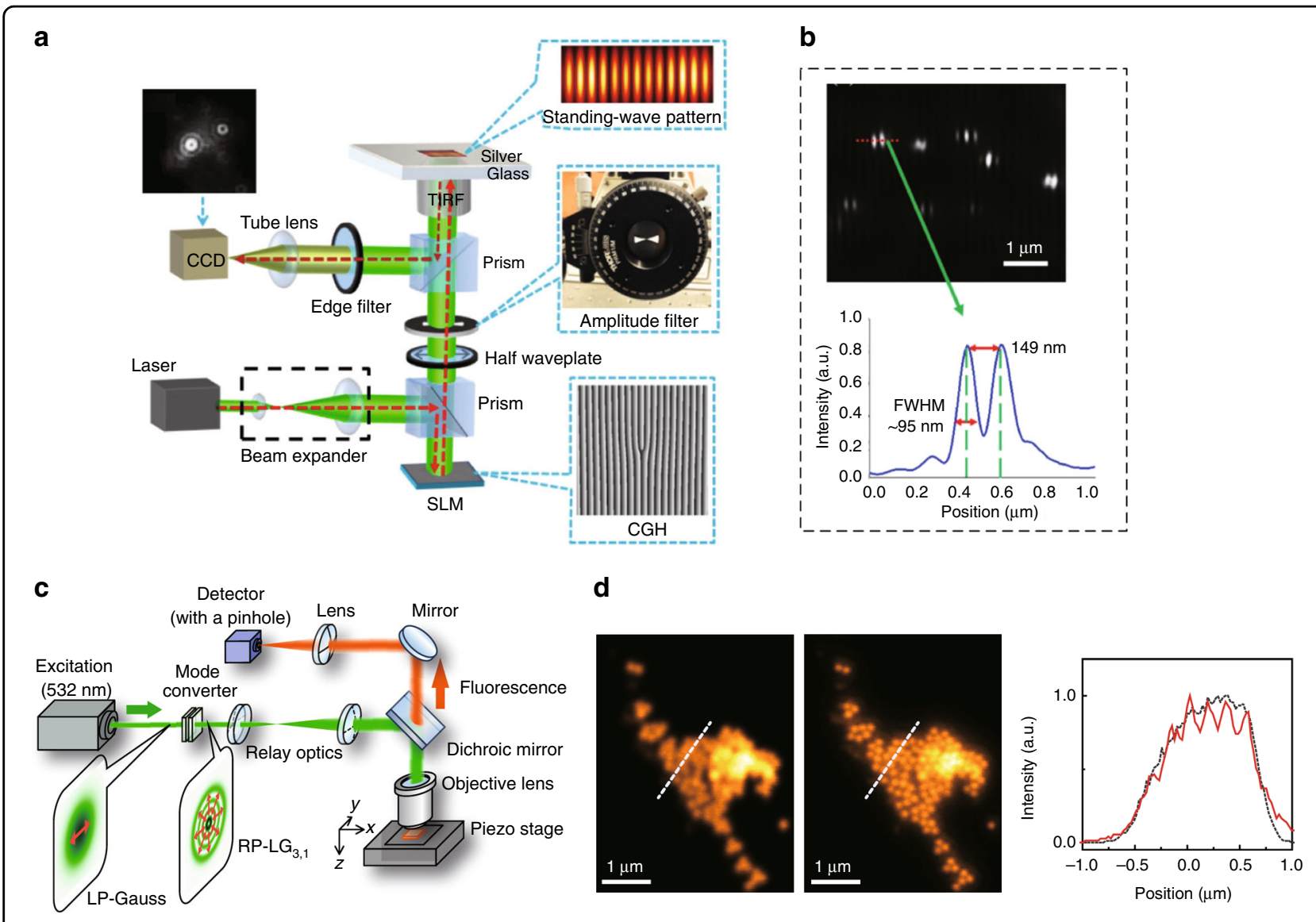

Fig. 18 High-resolution imaging using OVs. a Setup of the plasmonic structured illumination microscopy technique, and $\mathbf{b}$ imaging results with super-resolution ${ }^{277}$. c Setup of superoscillation focusing imaging using a vector VB, and $\mathbf{d}$ imaging results with super-resolution ${ }^{278}$. $\mathbf{a}, \mathbf{b}$ Reprinted with permission from ref. ${ }^{277}$, OSA Publishing. c, d Reprinted with permission from ref. ${ }^{278}$, OSA Publishing

enhance the excitation efficiency and reduce the background noise ${ }^{275}$. With the development of multisingularity beams, a vortex array was used to harness the point-spread function to realize high-resolution farfield microscopy ${ }^{276}$. Specifically, fractional VBs were also used for precise microscopy to reach sub-100-nm resolution $^{277}$. With the advanced vector VBs having a special polarization structure, the super-resolution imaging reached an even higher resolution ${ }^{278}$, as shown in Fig. 18c, d. With the quantum properties of VBs, quantum ghost imaging was combined with twisted photons, opening new routes for imaging techniques ${ }^{243}$. As a remarkable breakthrough of microscopy using OVs, the stimulated emission depletion (STED) microscopy technique proposed by Willig et al. ${ }^{279}$, in which the vortex phase is modulated in STED beams to realize super-resolution, was awarded the 2014 Nobel Prize in Chemistry.

\section{Biomedicine and chemistry}

Using OV tweezers, one can manipulate and assemble some proteins and other biomolecules, greatly advancing the development of structural chemistry and biomedical photonics ${ }^{34,36}$. Note that VBs and some organic molecules all have chirality, and the chirality of the vortex phase can interplay with that of a biomolecule, which has promoted a number of applications in biomedicine and chemistry $^{280}$. For instance, VBs can be used to assemble DNA ${ }^{36}$ and resolve enantiomers ${ }^{281}$ due to the chirality coupling effect. By applying this method to chiral metamaterials, novel sensing technology was proposed to detect many enantiomers or biomolecules, such as amino acids, sugars, and nucleotides ${ }^{282}$. Additionally, the functionalities of transporting subcellular organelles and exerting less photodamage on the trapped particle was developed for vortex tweezers, which have been used in sophisticated single-cell nanosurgery ${ }^{283}$. The advanced microscopy brought about by VBs was also used for observing biological cell structures with high resolution ${ }^{279}$. Most recently, vortices were directly generated from organic materials ${ }^{284}$, with further development of organic illumination and chemical detection technologies expected in the future. 


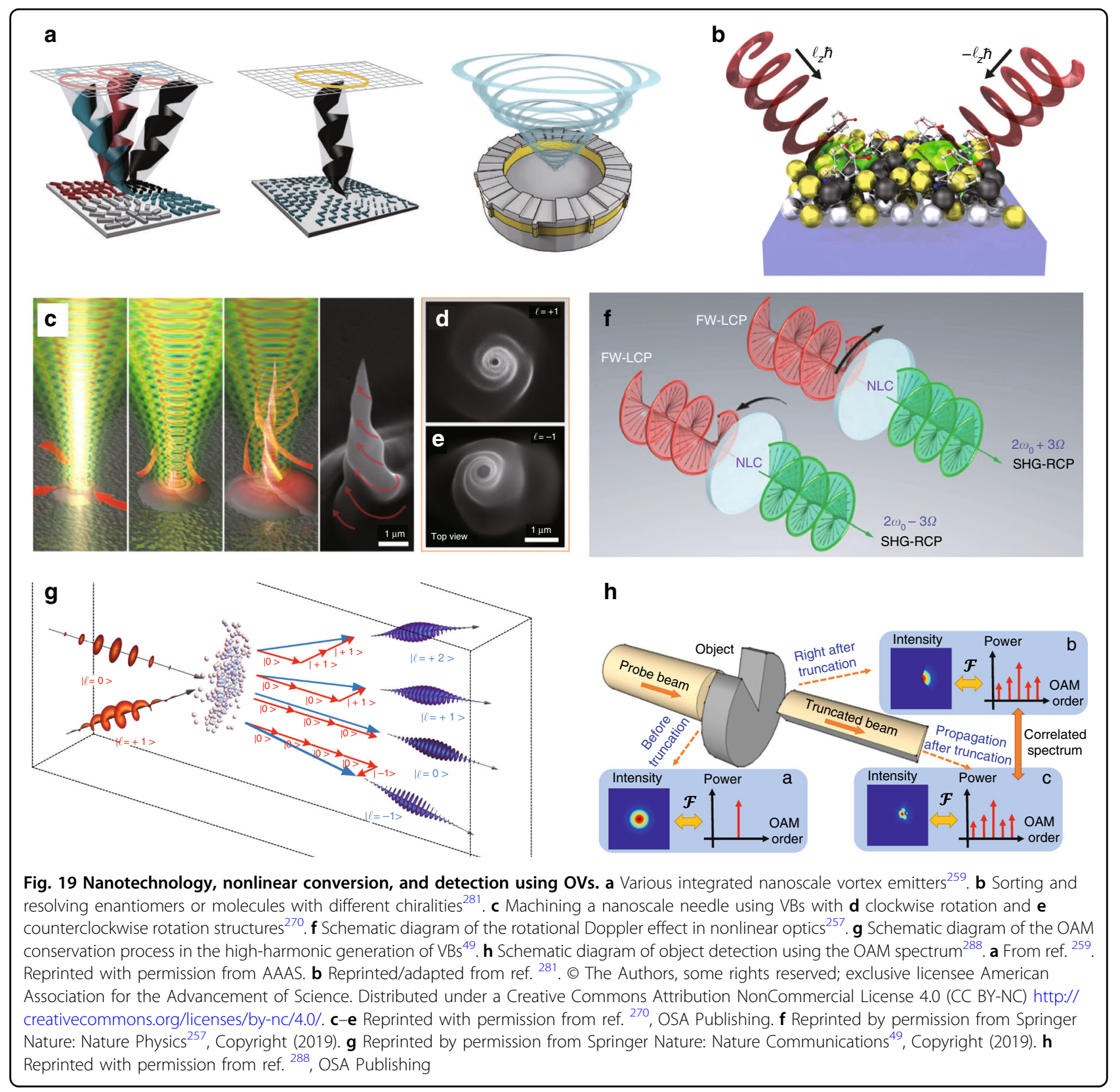

\section{Metrology}

Based on the light-matter interaction through which the OAM of light can be coupled with the mechanical momentum, VBs can be used to detect object motion, including spin motion ${ }^{285}$ and lateral motion ${ }^{286}$. With recent advances in nanophotonics and nanofabrication, the precision of detection has reached the nanoscale, and VBs can be used for label-free single-molecule detection in metamaterials $^{287}$. Recently, the OAM spectrum, acting as a new powerful tool, was used in optical detection, in which the difference between the OAM spectra of incident and outgoing light revealed the topography of the $\operatorname{target}^{288}$, as depicted in Fig. 19h. Similar OAM-spectrum methods have been successfully applied to detect complicated turbulence in the atmosphere ${ }^{289}$ and ocean ${ }^{290}$. Recently, with the study of the interaction between OVs and plasmonic nanoslits ${ }^{291}$, VBs have been used to detect the nanostructure on metal films, opening the door for onchip compact OAM detection $^{292}$. There are also several devices and structures for detecting OAM states. For instance, a virtual rotational antenna structure was designed to generate the rotational Doppler effect, and the signal of the Doppler shift could be detected to reveal the OAM of the corresponding $\mathrm{OV}^{293}$. The on-chip plasmonic nanoslit structure can produce different scattering effects for OVs with different TCs, serving as a useful tool for the discrimination of $\mathrm{OAM}^{294}$. 


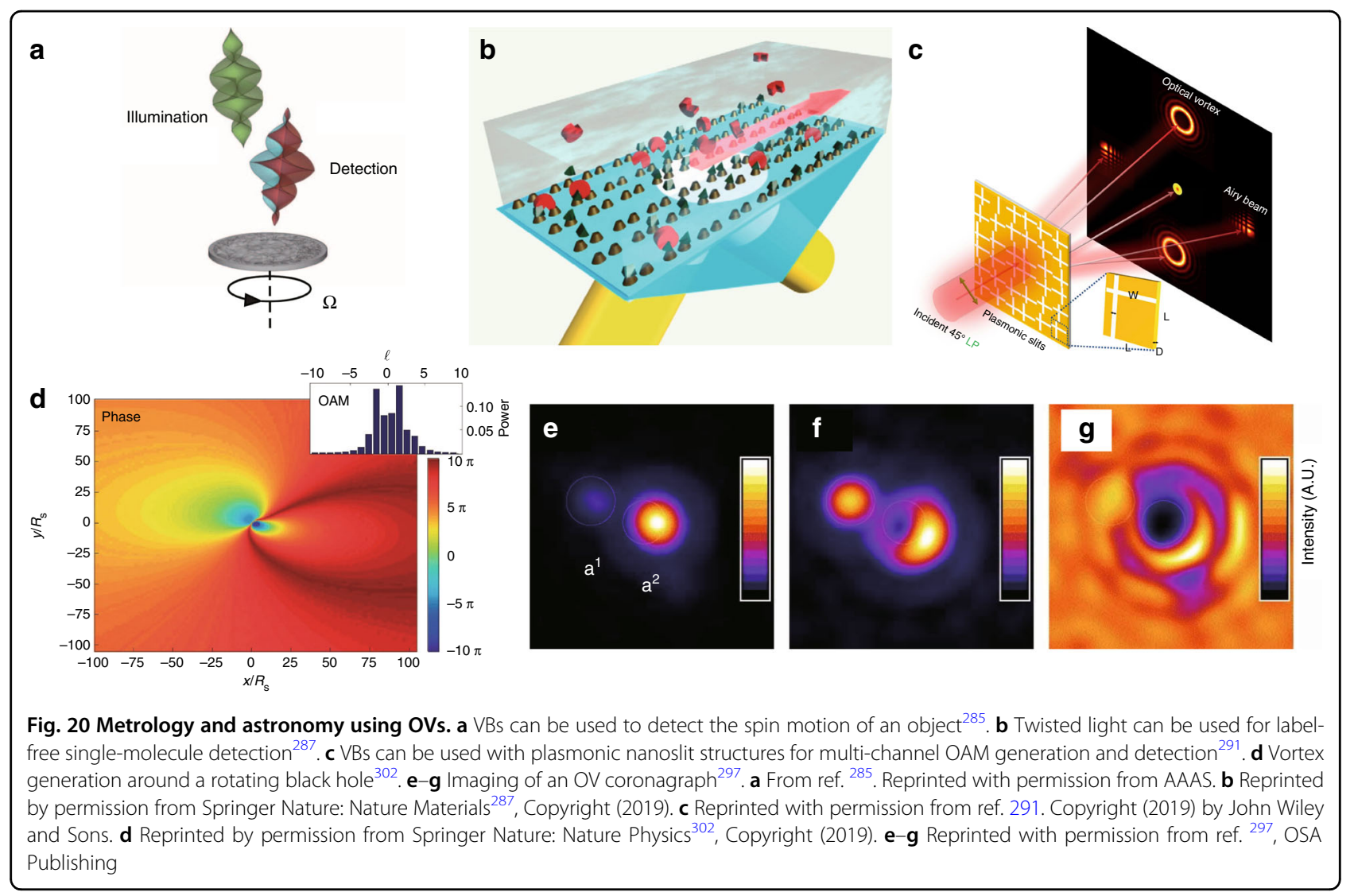

Moreover, some on-demand metasurface ${ }^{262}$ and liquid crystal $^{170,171,265}$ devices have shown great potential for detecting OAM, enabling the further development of precise metrology technologies.

\section{Astronomy}

OVs not only have been artificially created in laser beams but also naturally exist in the cosmic microwave background $^{35}$. In 2003, Harwit described astrophysical processes of OAM light generation, including photon scattering and vortex generation in the environments surrounding energetic sources, e.g., masers, pulsars, and quasars $^{35}$. To make an astronomical survey that took advantage of OVs, an OV coronagraph was designed ${ }^{295}$ and experimentally verified ${ }^{296}$ by Swartzlander's group, which has made many breakthroughs in astronomical demonstration ${ }^{297}$. In addition to the scalar vortex masks used in these coronagraph devices, vectorial masks were also implemented in coronagraphs at nearly the same time as Swartzlander's work in $2005^{298}$. With the development of vector OVs, the vortex coronagraph implemented in international ground-based telescope facilities has been based on vectorial vortex masks to obtain higher sensitivity and lower aberrations ${ }^{299}$. With the recent development of multi-singularity tunability, adaptive multiple-vortex coronagraph masks have been developed for multiple-star detections ${ }^{300,301}$. In 2011, Tamburini et al. ${ }^{302}$ reported the OAM light effect around rotating black holes, which provided a new method to detect black holes, as shown in Fig. 20d. Interestingly, astronomical applications are always accompanied by sci-fi themes, and vortex light has been declared to be a fast, furious and perfect tool for talking to aliens and detecting alien civilizations due to its unique properties ${ }^{303}$.

\section{Other advances}

OVs indeed demonstrate various characteristics, not only as VBs analysed under the paraxial approximation but also as a general spatial singular field with fractality of singularities. In addition, OVs are not restricted to linear space but have been extensively studied in nonlinear media in connection with optical solitons ${ }^{7,22-24}$. Moreover, topological vortex waves can be studied in other spectra in addition to the light field, such as microwave vortices $^{304}$, acoustic vortices ${ }^{305}$ and X-ray vortices ${ }^{50}$. Vortex electron beams ${ }^{59}$ and neutron beams ${ }^{60}$ with unique OAM properties were also produced and investigated. Very recently, gravitational waves with AM were observed and could be used for trapping and guiding cosmic bodies $^{306}$. Overall, there are currently numerous promising and amazing applications related to OVs with unlimited possibilities that require further exploration. 


\section{Conclusions and perspectives}

This review article is dedicated to commemorating the 30th anniversary of the birth of OVs, covering the development history from fundamental theories to tunable vortex techniques and then to widespread scientific applications. We first reviewed the theoretical foundation of OVs and emphasized the unique properties related to OAM, TC, and singularities. Then, we reviewed the recent advances in tunable VBs, where the tunability includes not only wavelength tunability and temporal tunability but also OAM tunability. Recent vortex generation methods with different kinds of tunability were reviewed, revealing the development of optical field manipulation. Taking advantage of the advanced vortex manipulation techniques, widespread novel applications have boomed in the new century. We reviewed the various applications in different branches of science as comprehensively as possible. The development tendency of OVs is a typical example that theories guide new applications and that application demands inspire new theories. To date, OVs are still hot topics and have high potential for both theories and applications.

\section{Acknowledgements}

This work was funded by The National Key Research and Development Program of China (Grant No. 2017YFB1104500), Natural Science Foundation of Beijing Municipality (4172030), Beijing Young Talents Support Project (2017000020124G044), Leading talents of Guangdong province program (00201505), National Natural Science Foundation of China (U1701661, 91750205, 61975133, 11604218, 61975087), and Natural Science Foundation of Guangdong Province (2016A030312010, 2017A030313351). The first author Y.S. would like to thank Prof. Andrew Forbes at Wits University for useful discussions and Xilin Yang at the Beijing Institute of Technology for assistance with the graphics.

\section{Author details \\ ${ }^{1}$ Key Laboratory of Photonic Control Technology (Tsinghua University), Ministry of Education, 100084 Beijing, China. ${ }^{2}$ State Key Laboratory of Precision Measurement Technology and Instruments, Department of Precision Instrument, Tsinghua University, 100084 Beijing, China. ${ }^{3}$ National Engineering Laboratory for Public Safety Risk Perception and Control by Big Data (NEL- PSRPC), China Academy of Electronics and Information Technology of CETC, China Electronic Technology Group Corporation, 100041 Beijing, China. ${ }^{4}$ Nanophotonics Research Center, Shenzhen University, 518060 Shenzhen, China}

\section{Conflict of interest}

The authors declare that they have no conflict of interest.

Received: 13 March 2019 Revised: 4 August 2019 Accepted: 20 August 2019 Published online: 02 October 2019

\footnotetext{
References

1. Coullet, P., Gil, L. \& Rocca, F. Optical vortices. Opt. Commun. 73, 403-408 (1989)

2. Graham, R. \& Haken, H. Laserlight_first example of a second-order phase transition far away from thermal equilibrium. Z. Phys. 237, 31-46 (1970).

3. Aranson, I. S. \& Kramer, L. The world of the complex Ginzburg-Landau equation. Rev. Mod. Phys. 74, 99-143 (2002).
}

4. Coullet, P., Gil, L. \& Lega, J. Defect-mediated turbulence. Phys. Rev. Lett. 62 1619-1622 (1989).

5. Brambilla, M. et al. Transverse laser patterns. I. Phase singularity crystals. Phys. Rev. A 43, 5090-5113 (1991).

6. Brambilla, M. et al. Transverse laser patterns. II. Variational principle for pattern selection, spatial multistability, and laser hydrodynamics. Phys. Rev. A 43 , 5114-5120 (1991).

7. Rosanov, N. N., Fedorov, S. V. \& Shatsev, A. N. Curvilinear motion of multivortex laser-soliton complexes with strong and weak coupling. Phys. Rev. Lett. 95, 053903 (2005).

8. Genevet, P. et al. Bistable and addressable localized vortices in semiconductor lasers. Phys. Rev. Lett. 104, 223902 (2010).

9. Barland, S. et al. Observation of "true" optical vortices in a laser system. in Nonlinear Photonics and Novel Optical Phenomena (eds Chen, Z. G. \& Morandotti, R.) 195-205 (Springer, New York, NY, 2012).

10. Bazhenov, V. Y., Soskin, M. S. \& Vasnetsov, M. V. Screw dislocations in light wavefronts. J. Mod. Opt. 39, 985-990 (1992).

11. Crasovan, L. C., Malomed, B. A. \& Mihalache, D. Stable vortex solitons in the two-dimensional Ginzburg-Landau equation. Phys. Rev. E 63, 016605 (2000).

12. Mihalache, D. et al. Stable topological modes in two-dimensional GinzburgLandau models with trapping potentials. Phys. Rev. A 82, 023813 (2010).

13. Fedorov, S. V. et al. Topologically multicharged and multihumped rotating solitons in wide-aperture lasers with a saturable absorber. IEEE J. Quantum Electron. 39, 197-205 (2003).

14. Paulau, P. V. et al. Vortex solitons in lasers with feedback. Opt. Express 18, 8859-8866 (2010).

15. Mihalache, D. et al. Stable vortex tori in the three-dimensional cubic-quintic Ginzburg-Landau equation. Phys. Rev. Lett. 97, 073904 (2006).

16. Allen, L. et al. Orbital angular momentum of light and the transformation of Laguerre-Gaussian laser modes. Phys. Rev. A 45, 8185-8189 (1992).

17. Beijersbergen, M. W. et al. Astigmatic laser mode converters and transfer of orbital angular momentum. Opt. Commun. 96, 123-132 (1993).

18. Dennis, M. R., O'Holleran, K. \& Padgett, M. J. Singular optics: optical vortices and polarization singularities. Prog. Opt. 53, 293-363 (2009).

19. $\mathrm{He}, \mathrm{H}$. et al. Direct observation of transfer of angular momentum to absorptive particles from a laser beam with a phase singularity. Phys. Rev. Lett. 75, 826-829 (1995).

20. Gahagan, K. T. \& Swartzlander, G. A. Optical vortex trapping of particles. Opt. Lett. 21, 827-829 (1996).

21. Simpson, N. B., Allen, L. \& Padgett, M. J. Optical tweezers and optical spanners with Laguerre-Gaussian modes. J. Mod. Opt. 43, 2485-2491 (1996).

22. Swartzlander, G. A. Jr. \& Law, C. T. Optical vortex solitons observed in Kerr nonlinear media. Phys. Rev. Lett. 69, 2503-2506 (1992).

23. Tikhonenko, V., Christou, J. \& Luther-Daves, B. Spiraling bright spatial solitons formed by the breakup of an optical vortex in a saturable self-focusing medium. J. Opt. Soc. Am. B 12, 2046-2052 (1995).

24. Firth, W. J. \& Skryabin, D. V. Optical solitons carrying orbital angular momentum. Phys. Rev. Lett. 79, 2450-2453 (1997).

25. Dholakia, K. et al. Second-harmonic generation and the orbital angular momentum of light. Phys. Rev. A 54, R3742-R3745 (1996).

26. Courtial, J. et al. Second-harmonic generation and the conservation of orbital angular momentum with high-order Laguerre-Gaussian modes. Phys. Rev. A 56, 4193-4196 (1997).

27. Soskin, M. S. et al. Topological charge and angular momentum of light beams carrying optical vortices. Phys. Rev. A 56, 4064-4075 (1997).

28. Courtial, J. et al. Rotational frequency shift of a light beam. Phys. Rev. Lett. 81, 4828-4830 (1998)

29. Scheuer, J. \& Orenstein, M. Optical vortices crystals: spontaneous generation in nonlinear semiconductor microcavities. Science 285, 230-233 (1999).

30. Mair, A. et al. Entanglement of the orbital angular momentum states of photons. Nature 412, 313-316 (2001).

31. Molina-Terriza, G., Torres, J. P. \& Torner, L. Twisted photons. Nat. Phys. 3 305-310 (2007).

32. Paterson, L. et al. Controlled rotation of optically trapped microscopic particles. Science 292, 912-914 (2001).

33. MacDonald, M. P. et al. Creation and manipulation of three-dimensional optically trapped structures. Science 296, 1101-1103 (2002).

34. Grier, D. G. A revolution in optical manipulation. Nature 424, 810-816 (2003).

35. Harwit, M. Photon orbital angular momentum in astrophysics. Astrophys. J. 597, 1266-1270 (2003) 
36. Zhuang, X. W. Unraveling DNA condensation with optical tweezers. Science 305, 188-190 (2004).

37. Fürhapter, S. et al. Spiral phase contrast imaging in microscopy. Opt. Express 13, 689-694 (2005)

38. Tamburini, F. et al. Overcoming the Rayleigh criterion limit with optical vortices. Phys. Rev. Lett. 97, 163903 (2006).

39. Barreiro, J. T., Wei, T. C. \& Kwiat, P. G. Beating the channel capacity limit for linear photonic superdense coding. Nat. Phys. 4, 282-286 (2008).

40. Hickmann, J. M. et al. Unveiling a truncated optical lattice associated with a triangular aperture using light's orbital angular momentum. Phys. Rev. Lett. 105, 053904 (2010).

41. Yu, N. F. et al. Light propagation with phase discontinuities: generalized laws of reflection and refraction. Science 334, 333-337 (2011).

42. Cai, X. L. et al. Integrated compact optical vortex beam emitters. Science $\mathbf{3 3 8}$ 363-366 (2012).

43. Wang, J. et al. Terabit free-space data transmission employing orbital angular momentum multiplexing. Nat, Photonics 6, 488-496 (2012).

44. Bozinovic, N. et al. Terabit-scale orbital angular momentum mode division multiplexing in fibers. Science 340, 1545-1548 (2013).

45. Fickler, R. et al. Quantum entanglement of angular momentum states with quantum numbers up to 10,010. Proc. Natl Acad. Sci. USA 113, 13642-13647 (2016).

46. Devlin, R. C. et al. Arbitrary spin-to-orbital angular momentum conversion of light. Science 358, 896-901 (2017).

47. Stav, T. et al. Quantum entanglement of the spin and orbital angular momentum of photons using metamaterials. Science 361, 1101-1104 (2018).

48. Kong, F. Q. et al. Controlling the orbital angular momentum of high harmonic vortices. Nat. Commun. 8, 14970 (2017).

49. Gauthier, D. et al. Tunable orbital angular momentum in high-harmonic generation. Nat. Commun. 8, 14971 (2017).

50. Lee, J. C. T. et al. Laguerre-Gauss and Hermite-Gauss soft X-ray states generated using diffractive optics. Nat. Photonics 13, 205-209 (2019).

51. Xie, Z. W. et al. Ultra-broadband on-chip twisted light emitter for optical communications. Light Sci. Appl. 7, 18001 (2018).

52. Zambon, N. C. et al. Optically controlling the emission chirality of microlasers. Nat. Photonics 13, 283-288 (2019).

53. Rego, L. et al. Generation of extreme-ultraviolet beams with time-varying orbital angular momentum. Science 364, eaaw9486 (2019).

54. Nye, J. F. \& Berry, M. V. Dislocations in wave trains. Proc. R Soc A Math. Phys. Eng. Sci. 336, 165-190 (1974).

55. Penrose, L. S. \& Penrose, R. Impossible objects: a special type of visual illusion. Br. J. Psychol. 49, 31-33 (1958).

56. Bauer, T. et al. Observation of optical polarization Möbius strips. Science $\mathbf{3 4 7}$ 964-966 (2015).

57. Leach, J., Yao, E. \& Padgett, M. J. Observation of the vortex structure of a noninteger vortex beam. New J. Phys. 6, 71 (2004).

58. Berry, M. V. Optical vortices evolving from helicoidal integer and fractional phase steps. J. Opt. A Pure Appl. Opt. 6, 259-268 (2004).

59. Verbeeck, J., Tian, H. \& Schattschneider, P. Production and application of electron vortex beams. Nature 467, 301-304 (2010).

60. Clark, C. W. et al. Controlling neutron orbital angular momentum. Nature $\mathbf{5 2 5}$ 504-506 (2015).

61. Wang, X. W. et al. Recent advances on optical vortex generation. Nanophotonics 7, 1533-1556 (2018).

62. Zhu, L. \& Wang, J. A review of multiple optical vortices generation: methods and applications. Front. Optoelectron. 12, 52-68 (2019).

63. Chen, M. L. M., Jiang, L. J. \& Sha, W. E. I. Orbital angular momentum generation and detection by geometric-phase based metasurfaces. Appl. Sci. 8, 362 (2018).

64. Barnett, S. M., Babiker, M. \& Padgett, M. J. Optical orbital angular momentum. Philos. Trans. R Soc. A Math. Phys. Eng. Sci. 375, 20150444 (2017).

65. Padgett, M. J. Orbital angular momentum 25 years on [Invited]. Opt. Express 25, 11265-11274 (2017).

66. Yao, A. M. \& Padgett, M. J. Orbital angular momentum: origins, behavior and applications. Adv. Opti. Photonics 3, 161-204 (2011).

67. Milonni, P. W. \& Boyd, R. W. Momentum of light in a dielectric medium. Adv. Opt. Photonics 2, 519-553 (2010).

68. Nelson, D. F. Momentum, pseudomomentum, and wave momentum: toward resolving the Minkowski-Abraham controversy. Phys. Rev. A 44, 3985-3996 (1991).
69. Bliokh, K. Y., Bekshaev, A. Y. \& Nori, F. Optical momentum, spin, and angular momentum in dispersive media. Phys. Rev. Lett. 119, 073901 (2017).

70. Bliokh, K. Y. \& Nori, F. Transverse and longitudinal angular momenta of light. Phys. Rep. 592, 1-38 (2015).

71. Karimi, E. \& Boyd, R. W. Classical entanglement? Science 350, 1172-1173 (2015).

72. Chen, Y. F., Lu, T. H. \& Huang, K. F. Observation of spatially coherent polarization vector fields and visualization of vector singularities. Phys. Rev. Lett. 96, 033901 (2006)

73. Chen, Y. F. et al. Observation of vector vortex lattices in polarization states of an isotropic microcavity laser. Phys. Rev. Lett. 90, 053904 (2003).

74. Rosales-Guzmán, C., Ndagano, B. \& Forbes, A. A review of complex vector light fields and their applications. J. Opt. 20, 123001 (2018).

75. Abramochkin, E. \& Alieva, T. Closed-form expression for mutual intensity evolution of Hermite-Laguerre-Gaussian Schell-model beams. Opt. Lett. 42, 4032-4035 (2017).

76. Alieva, T. \& Bastiaans, M. J. Mode mapping in paraxial lossless optics. Opt. Lett. 30, 1461-1463 (2005).

77. Abramochkin, E. G. \& Volostnikov, V. G. Generalized Hermite-Laguerre-Gauss beams. Phys. Wave Phenom. 18, 14-22 (2010).

78. Bandres, M. A. \& Gutiérrez-Vega, J. C. Ince-Gaussian beams. Opt. Lett. 29, 144-146 (2004)

79. Bandres, M. A. \& Gutiérrez-Vega, J. C. Elliptical beams. Opt. Express 16, 21087-21092 (2008)

80. Bandres, M. A. \& Gutiérrez-Vega, J. C. Ince-Gaussian modes of the paraxial wave equation and stable resonators. J. Opt. Soc. Am. A 21, 873-880 (2004).

81. Bentley, J. B. et al. Generation of helical Ince-Gaussian beams with a liquidcrystal display. Opt. Lett. 31, 649-651 (2006).

82. Woerdemann, M., Alpmann, C. \& Denz, C. Optical assembly of microparticles into highly ordered structures using Ince-Gaussian beams. Appl. Phys. Lett. 98, 111101 (2011)

83. Shen, Y. J. et al. Hybrid topological evolution of multi-singularity vortex beams: generalized nature for helical-Ince-Gaussian and Hermite-LaguerreGaussian modes. J. Opt. Soc. Am. A 36, 578-587 (2019).

84. Volke-Sepulveda, K. et al. Orbital angular momentum of a high-order Besse light beam. J. Opt. B Quantum Semiclassical Opt. 4, S82-S89 (2002).

85. Gutiérrez-Vega, J. C., Iturbe-Castillo, M. D. \& Chávez-Cerda, S. Alternative formulation for invariant optical fields: Mathieu beams. Opt. Lett. 25, 1493-1495 (2000).

86. Lóxpez-Mariscal, C. et al. Orbital angular momentum transfer in helical Mathieu beams. Opt. Express 14, 4182-4187 (2006).

87. Chávez-Cerda, S. et al. Holographic generation and orbital angular momentum of high-order Mathieu beams. J. Opt. B Quantum Semiclassical Opt. 4, S52-S57 (2002).

88. Alpmann, $C$. et al. Mathieu beams as versatile light moulds for 3D micro particle assemblies. Opt. Express 18, 26084-26091 (2010).

89. Zhu, L. \& Wang, J. Demonstration of obstruction-free data-carrying $\mathrm{N}$-fold Bessel modes multicasting from a single Gaussian mode. Opt. Lett. 40, 5463-5466 (2015).

90. Bužek, V. \& Quang, T. Generalized coherent state for bosonic realization of SU (2)Lie algebra. J. Opt. Soc. Am. B 6, 2447-2449 (1989).

91. Lin, Y. C. et al. Model of commensurate harmonic oscillators with SU(2) coupling interactions: Analogous observation in laser transverse modes. Phys. Rev. E 85, 046217 (2012).

92. Tuan, P. H. et al. Realizing high-pulse-energy large-angular-momentum beams by astigmatic transformation of geometric modes in an $\mathrm{Nd}: \mathrm{YAG} / \mathrm{Cr}^{4+}$ : YAG laser. IEEE J. Sel. Top. Quantum Electron. 24, 1600809 (2018).

93. Tung, J. C. et al. Exploring vortex structures in orbital-angular-momentum beams generated from planar geometric modes with a mode converter. Opt. Express 24, 22796-22805 (2016).

94. Chen, Y. F. et al. Devil's staircase in three-dimensional coherent waves localized on Lissajous parametric surfaces. Phys. Rev. Lett. 96, 213902 (2006).

95. Lu, T. H. et al. Three-dimensional coherent optical waves localized on trochoidal parametric surfaces. Phys. Rev. Lett. 101, 233901 (2008).

96. Shen, Y. J. et al. Polygonal vortex beams. IEEE Photonics J. 10, 1503016 (2018).

97. Shen, Y. J., Fu, X. \& Gong, M. L. Truncated triangular diffraction lattices and orbital-angular-momentum detection of vortex SU(2) geometric modes. Opt. Express 26, 25545-25557 (2018).

98. Freund, I. Optical Möbius strips in three-dimensional ellipse fields: I. Lines of circular polarization. Opt. Commun. 283, 1-15 (2010). 
99. Freund, I. Optical Möbius strips in three dimensional ellipse fields: II. Lines of linear polarization. Opt. Commun. 283, 16-28 (2010).

100. Galvez, E. J. et al. Multitwist Möbius strips and twisted ribbons in the polarization of paraxial light beams. Sci. Rep. 7, 13653 (2017).

101. Veretenov, N. A., Fedorov, S. V. \& Rosanov, N. N. Topological vortex and knotted dissipative optical 3D solitons generated by 2D vortex solitons. Phys. Rev. Lett. 119, 263901 (2017).

102. Leach, J. et al. Vortex knots in light. New J. Phys. 7, 55 (2005).

103. Kleckner, D. \& Irvine, W. T. M. Creation and dynamics of knotted vortices. Nat. Phys. 9, 253-258 (2013).

104. Dennis, M. R. et al. Isolated optical vortex knots. Nat. Phys. 6, 118-121 (2010).

105. Tempone-Wiltshire, S. J., Johnstone, S. P. \& Helmerson, K. Optical vortex knots-one photon at a time. Sci. Rep. 6, 24463 (2016).

106. Cunzhi, S., Pu, J. X. \& Chávez-Cerda, S. Elegant Cartesian Laguerre-Hermite-Gaussian laser cavity modes. Opt. Lett. 40, 1105-1108 (2015).

107. Ellenbogen, T. et al. Nonlinear generation and manipulation of Airy beams. Nat. Photonics 3, 395-398 (2009).

108. Ring, J. D. et al. Auto-focusing and self-healing of Pearcey beams. Opt. Express 20, 18955-18966 (2012).

109. Bandres, M. A., Gutiérrez-Vega, J. C. \& Chávez-Cerda, S. Parabolic nondiffracting optical wave fields. Opt. Lett. 29, 44-46 (2004).

110. O'Holleran, K. et al. Fractality of light's darkness. Phys. Rev. Lett. 100, 053902 (2008).

111. Zhang, L. G. et al. Deflection of a reflected intense vortex laser beam. Phys. Rev. Lett. 117, 113904 (2016).

112. Omatsu, T., Miyamoto, K. \& Lee, A. J. Wavelength-versatile optical vortex lasers. J. Opt. 19, 123002 (2017).

113. de Araujo, L. E. E. \& Anderson, M. E. Measuring vortex charge with a triangular aperture. Opt. Lett. 36, 787-789 (2011).

114. Mourka, A. et al. Visualization of the birth of an optical vortex using diffraction from a triangular aperture. Opt. Express 19, 5760-5771 (2011).

115. Melo, L. A. et al. Direct measurement of the topological charge in elliptical beams using diffraction by a triangular aperture. Sci. Rep. 8, 6370 (2018).

116. Ghai, D. P., Senthilkumaran, P. \& Sirohi, R. S. Single-slit diffraction of an optical beam with phase singularity. Opt. Lasers Eng. 47, 123-126 (2009).

117. Mesquita, P. H. F. et al. Engineering a square truncated lattice with light's orbital angular momentum. Opt. Express 19, 20616-20621 (2011).

118. Liu, Y. X. et al. Propagation of an optical vortex beam through a diamondshaped aperture. Opt. Laser Technol. 45, 473-479 (2013).

119. Ambuj, A., Vyas, R. \& Singh, S. Diffraction of orbital angular momentum carrying optical beams by a circular aperture. Opt. Lett. 39, 5475-5478 (2014).

120. Taira, Y. \& Zhang, S. K. Split in phase singularities of an optical vortex by offaxis diffraction through a simple circular aperture. Opt. Lett. 42, 1373-1376 (2017).

121. Bahl, M. \& Senthilkumaran, P. Energy circulations in singular beams diffracted through an isosceles right triangular aperture. Phys. Rev. A 92, 013831 (2015).

122. Chen, R. S. et al. Detecting the topological charge of optical vortex beams using a sectorial screen. Appl. Opt. 56, 4868-4872 (2017).

123. Zhang, W. H. et al. Experimental demonstration of twisted light's diffraction theory based on digital spiral imaging. Chin. Opt. Lett. 14, 110501 (2016).

124. Ram, B. S. B., Sharma, A. \& Senthilkumaran, P. Diffraction of V-point singularities through triangular apertures. Opt. Express 25, 10270-10275 (2017).

125. Holleczek, A. et al. Classical and quantum properties of cylindrically polarized states of light. Opt. Express 19, 9714-9736 (2011).

126. Milione, G. et al. Higher-order Poincaré sphere, Stokes parameters, and the angular momentum of light. Phys. Rev. Lett. 107, 053601 (2011).

127. Naidoo, D. et al. Controlled generation of higher-order Poincaré sphere beams from a laser. Nat. Photonics 10, 327-332 (2016).

128. Yi, X. N. et al. Hybrid-order Poincaré sphere. Phys. Rev. A 91, 023801 (2015).

129. Liu, Z. X. et al. Generation of arbitrary vector vortex beams on hybrid-order Poincaré sphere. Photonics Res. 5, 15-21 (2017).

130. Wang, R. S. et al. Electrically driven generation of arbitrary vector vortex beams on the hybrid-order Poincaré sphere. Opt. Lett. 43, 3570-3573 (2018).

131. Franke-Arnold, S. et al. Uncertainty principle for angular position and angular momentum. New J. Phys. 6, 103 (2004).

132. Leach, J. et al. Quantum correlations in optical angle-orbital angular momentum variables. Science 329, 662-665 (2010).

133. Jha, A. K. et al. Fourier relationship between the angle and angular momentum of entangled photons. Phys. Rev. A 78, 043810 (2008).
134. Erhard, M. et al. Twisted photons: new quantum perspectives in high dimensions. Light Sci. Appl. 7, 17146 (2018).

135. Otte, E. et al. Entanglement beating in free space through spin-orbit coupling. Light Sci. Appl. 7, 18009 (2018).

136. Bliokh, K. Y. et al. Spin-orbit interactions of light. Nat. Photonics 9, 796-808 (2015).

137. Cardano, F. \& Marrucci, L. Spin-orbit photonics. Nat. Photonics 9, 776-778 (2015).

138. Shao, Z. K. et al. Spin-orbit interaction of light induced by transverse spin angular momentum engineering. Nat. Commun. 9, 926 (2018).

139. Magaña-Loaiza, O. S. et al. Hanbury brown and Twiss interferometry with twisted light. Sci. Adv. 2, e1501143 (2016).

140. Mohanty, A. et al. Quantum interference between transverse spatial waveguide modes. Nat. Commun. 8, 14010 (2017).

141. Zhang, Y. W. et al. Engineering two-photon high-dimensional states through quantum interference. Sci. Adv. 2, e1501165 (2016).

142. Yin, X. B. et al. Photonic spin Hall effect at metasurfaces. Science 339, 1405-1407 (2013).

143. Liu, Y. C. et al. Photonic spin Hall effect in metasurfaces: a brief review. Nanophotonics 6, 51-70 (2017).

144. Forbes, A., Dudley, A. \& McLaren, M. Creation and detection of optical modes with spatial light modulators. Adv. Opt. Photonics 8, 200-227 (2016).

145. Berkhout, G. C. G. et al. Efficient sorting of orbital angular momentum states of light. Phys. Rev. Lett. 105, 153601 (2010).

146. Wen, Y. H. et al. Spiral transformation for high-resolution and efficient sorting of optical vortex modes. Phys. Rev. Lett. 120, 193904 (2018).

147. Liu, G. G. et al. Measurement of the topological charge and index of vortex vector optical fields with a space-variant half-wave plate. Opt. Lett. 43, 823-826 (2018).

148. Ndagano, B. et al. Beam quality measure for vector beams. Opt. Lett. 41 3407-3410 (2016).

149. McLaren, M., Konrad, T. \& Forbes, A. Measuring the nonseparability of vector vortex beams. Phys. Rev. A 92, 023833 (2015).

150. Forbes, A. Controlling light's helicity at the source: orbital angular momentum states from lasers. Philos. Trans. R Soc. A Math. Phys. Eng. Sci. 375, 20150436 (2017).

151. Qiao, Z. et al. Generating high-charge optical vortices directly from laser up to 288th order. Laser Photonics Rev. 12, 1800019 (2018).

152. Lee, C. Y. et al. Generation of higher order vortex beams from a $\mathrm{WO} 4 / \mathrm{Nd}$ : WO4 self-Raman laser via off-axis pumping with mode converter. IEEE J. Sel. Top Quantum Electron. 21, 1600305 (2015).

153. Sueda, K. et al. Laguerre-Gaussian beam generated with a multilevel spiral phase plate for high intensity laser pulses. Opt. Express 12, 3548-3553 (2004).

154. Cardano, F. et al. Polarization pattern of vector vortex beams generated by $q^{-}$ plates with different topological charges. Appl. Opt. 51, C1-C6 (2012).

155. Marrucci, L. The q-plate and its future. J. Nanophotonics 7, 078598 (2013).

156. Brasselet, E. Tunable high-resolution macroscopic self-engineered geometric phase optical elements. Phys. Rev. Lett. 121, 033901 (2018).

157. Mock, A., Sounas, D. \& Alù, A. Tunable orbital angular momentum radiation from angular-momentum-biased microcavities. Phys. Rev. Lett. 121, 103901 (2018).

158. Zhou, N. et al. Generating and synthesizing ultrabroadband twisted light using a compact silicon chip. Opt. Lett. 43, 3140-3143 (2018).

159. Horikawa, M. T. et al. Handedness control in a tunable midinfrared (6.0 $12.5 \mu \mathrm{m})$ vortex laser. J. Opt. Soc. Am. B 32, 2406-2410 (2015).

160. Abulikemu, A. et al. Widely-tunable vortex output from a singly resonant optical parametric oscillator. Opt. Express 23, 18338-18344 (2015).

161. Zhang, W. D. et al. Optical vortex generation with wavelength tunability based on an acoustically-induced fiber grating. Opt. Express 24, 19278-19285 (2016).

162. Lyubopytov, V. S. et al. Simultaneous wavelength and orbital angular momentum demultiplexing using tunable MEMS-based Fabry-Perot filter. Opt. Express 25, 9634-9646 (2017).

163. Liu, Q. Y. et al. Wavelength- and OAM-tunable vortex laser with a reflective volume Bragg grating. Opt. Express 25, 23312-23319 (2017).

164. Yao, S. Z. et al. Tunable orbital angular momentum generation using all-fiber fused coupler. IEEE Photonics Technol. Lett. 30, 99-102 (2018).

165. Shen, Y. J. et al. Wavelength-tunable Hermite-Gaussian modes and an orbital-angular-momentum-tunable vortex beam in a dual-off-axis pumped Yb:CALGO laser. Opt. Lett. 43, 291-294 (2018). 
166. Shen, Y. J. et al. Dual-wavelength vortex beam with high stability in a diodepumped Yb:CaGdAlO 4 laser. Laser Phys. Lett. 15, 055803 (2018).

167. Wang, S. et al. Generation of wavelength- and OAM-tunable vortex beam at low threshold. Opt. Express 26, 18164-18170 (2018).

168. Zhou, N., Liu, J. \& Wang, J. Reconfigurable and tunable twisted light laser. Sci. Rep. 8, 11394 (2018).

169. Fadeyeva, T. A. et al. Spatially engineered polarization states and optical vortices in uniaxial crystals. Opt. Express 18, 10848-10863 (2010).

170. Rafayelyan, M., Tkachenko, G. \& Brasselet, E. Reflective spin-orbit geometric phase from chiral anisotropic optical media. Phys. Rev. Lett. 116, 253902 (2016).

171. Kobashi, J., Yoshida, H. \& Ozaki, M. Polychromatic optical vortex generation from patterned cholesteric liquid crystals. Phys. Rev. Lett. 116, 253903 (2016).

172. Piccirillo, B. et al. Photon spin-to-orbital angular momentum conversion via an electrically tunable q-plate. Appl. Phys. Lett. 97, 241104 (2010).

173. Toyoda, K. et al. Using optical vortex to control the chirality of twisted metal nanostructures. Nano Lett. 12, 3645-3649 (2012).

174. Yang, L. et al. Direct laser writing of complex microtubes using femtosecond vortex beams. Appl. Phys. Lett. 110, 221103 (2017)

175. Zürch, M. et al. Strong-field physics with singular light beams. Nat. Phys. 8, 743-746 (2012).

176. Ran, L. L., Guo, Z. Y. \& Qu, S. L. Rotational motions of optically trapped microscopic particles by a vortex femtosecond laser. Chin. Phys. B 21, 104206 (2012).

177. Ishaaya, A. A. et al. Efficient selection of high-order Laguerre-Gaussian modes in a Q-switched Nd:YAG laser. IEEE J. Quantum Electron. 39, 74-82 (2003).

178. Kim, D. J., Kim, J. W. \& Clarkson, W. A. Q-switched Nd:YAG optical vortex lasers. Opt. Express 21, 29449-29454 (2013).

179. Zhao, Y. G. et al. $1 \mathrm{~mJ}$ pulsed vortex laser at $1645 \mathrm{~nm}$ with well-defined helicity. Opt. Express 24, 15596-15602 (2016).

180. Chang, C. C. et al. Generating high-peak-power structured lights in selectively pumped passively Q-switched lasers with astigmatic mode transformations. Laser Phys. 27, 125805 (2017)

181. He, H. S. et al. Low-threshold, nanosecond, high-repetition-rate vortex pulses with controllable helicity generated in $\mathrm{Cr}$, Nd:YAG self-Q-switched microchip laser. Laser Phys. 28, 055802 (2018).

182. Wang, Y. B. et al. Generation of $1535-\mathrm{nm}$ pulsed vortex beam in a diodepumped Er, Yb:glass microchip laser. IEEE Photonics Technol. Lett. 30, 891-894 (2018)

183. Koyama, M. et al. Power scaling of a picosecond vortex laser based on a stressed Yb-doped fiber amplifier. Opt. Express 19, 994-999 (2011).

184. Liang, H. C. et al. Compact efficient multi-GHz Kerr-lens mode-locked diodepumped Nd: $\mathrm{YO}_{4}$ laser. Opt. Express 16, 21149-21154 (2008).

185. Liang, H. C. et al. Picosecond optical vortex converted from multigigahertz self-mode-locked high-order Hermite-Gaussian Nd:GdVO 4 lasers. Opt. Letters 34, 3842-3844 (2009).

186. Liang, H. C. et al. Total self-mode locking of multi-pass geometric modes in diode-pumped Nd:WO $\mathrm{WO}_{4}$ lasers. Laser Phys. Lett. 10, 105804 (2013).

187. Tung, J. C. et al. Exploring the self-mode locking and vortex structures of nonplanar elliptical modes in selectively end-pumped $\mathrm{Nd} \mathrm{WO}_{4}$ lasers: manifestation of large fractional orbital angular momentum. Opt. Express 25, 22769-22779 (2017).

188. Huang, K. et al. Controlled generation of ultrafast vector vortex beams from a mode-locked fiber laser. Opt. Lett. 43, 3933-3936 (2018).

189. Bolze, T. \& Nuernberger, P. Temporally shaped Laguerre-Gaussian femtosecond laser beams. Appl. Opt. 57, 3624-3628 (2018).

190. Zhuang, W. Z. et al. High-power high-repetition-rate subpicosecond monolithic Yb:KGW laser with self-mode locking. Opt. Lett. 38, 2596-2599 (2013).

191. Chang, M. T. et al. Exploring transverse pattern formation in a dualpolarization self-mode-locked monolithic Yb: KGW laser and generating a 25$\mathrm{GHz}$ sub-picosecond vortex beam via gain competition. Opt. Express $\mathbf{2 4}$ 8754-8762 (2016).

192. Zhang, Z. M. et al. Generation of all-fiber femtosecond vortex laser based on NPR mode-locking and mechanical LPG. Chin. Opt. Lett. 16, 110501 (2018).

193. Wang, S. et al. Direct emission of chirality controllable femtosecond $L G_{01}$ vortex beam. Appl. Phys. Lett. 112, 201110 (2018).

194. Wang, S. et al. Direct generation of femtosecond vortex beam from a Yb:KYW oscillator featuring a defect-spot mirror. OSA Contin. 2, 523-530 (2019).

195. Woerdemann, M. et al. Advanced optical trapping by complex beam shaping. Laser Photonics Rev. 7, 839-854 (2013).
196. Li, X. F. et al. Automultiscopic displays based on orbital angular momentum of light. J. Opt. 18, 085608 (2016)

197. Anguita, J. A., Herreros, J. \& Djordjevic, I. B. Coherent multimode OAM superpositions for multidimensional modulation. IEEE Photonics J. 6, 7900811 (2014).

198. Padgett, M. et al. An experiment to observe the intensity and phase structure of Laguerre-Gaussian laser modes. Am. J. Phys. 64, 77-82 (1996).

199. Courtial, J. \& Padgett, M. J. Performance of a cylindrical lens mode converter for producing Laguerre-Gaussian laser modes. Opt. Commun. 159, 13-18 (1999).

200. O'Neil, A. T. \& Courtial, J. Mode transformations in terms of the constituent Hermite-Gaussian or Laguerre-Gaussian modes and the variable-phase mode converter. Opt. Commun. 181, 35-45 (2000).

201. Padgett, M. J. \& Allen, L. Orbital angular momentum exchange in cylindrical-lens mode converters. J. Opt. B Quantum Semiclassical Opt. 4 S17-S19 (2002).

202. Shen, Y. J. et al. Observation of spectral modulation coupled with broadband transverse-mode-locking in an Yb:CALGO frequency-degenerate cavity. Chin. Opt. Lett. 17, 031404 (2019).

203. Shen, Y. J. et al. Vortex lattices with transverse-mode-locking states switching in a large-aperture off-axis-pumped solid-state laser. J. Opt. Soc. Am. B 35 2940-2944 (2018).

204. Shen, Y. J. et al. Periodic-trajectory-controlled, coherent-state-phase-switched, and wavelength-tunable SU(2) geometric modes in a frequency-degenerate resonator. Appl. Opt. 57, 9543-9549 (2018).

205. Ngcobo, S. et al. A digital laser for on-demand laser modes. Nat. Commun. 4 2289 (2013).

206. Porfirev, A. P. \& Khonina, S. N. Simple method for efficient reconfigurable optical vortex beam splitting. Opt. Express 25, 18722-18735 (2017).

207. Ma, H. X. et al. Generation of circular optical vortex array. Ann. Phys. $\mathbf{5 2 9}$ 1700285 (2017).

208. Li, L. et al. Generation of optical vortex array along arbitrary curvilinear arrangement. Opt. Express 26, 9798-9812 (2018).

209. Wan, Z. S. et al. Quadrant-separable multi-singularity vortices manipulation by coherent superposed mode with spatial-energy mismatch. Opt. Express $\mathbf{2 6}$ 34940-34955 (2018).

210. Hou, T. Y. et al. Spatially-distributed orbital angular momentum beam array generation based on greedy algorithms and coherent combining technology. Opt. Express 26, 14945-14958 (2018).

211. Gbur, G. Fractional vortex Hilbert's hotel. Optica 3, 222-225 (2016).

212. Wang, Y. Y. D. \& Gbur, G. Hilbert's Hotel in polarization singularities. Opt. Lett. 42, 5154-5157 (2017).

213. Ferrando, A. \& García-March, M. A. Analytical solution for multi-singular vortex Gaussian beams: the mathematical theory of scattering modes. J. Opt. $\mathbf{1 8}$ 064006 (2016).

214. Brasselet, E. Tunable optical vortex arrays from a single nematic topological defect. Phys. Rev. Lett. 108, 087801 (2012).

215. Barboza, R. et al. Harnessing optical vortex lattices in nematic liquid crystals. Phys. Rev. Lett. 111, 093902 (2013).

216. Ashkin, A. Acceleration and trapping of particles by radiation pressure. Phys. Rev. Lett. 24, 156-159 (1970).

217. Padgett, M. \& Bowman, R. Tweezers with a twist. Nat. Photonics 5, 343-348 (2011)

218. Chapin, S. C., Germain, V. \& Dufresne, E. R. Automated trapping, assembly, and sorting with holographic optical tweezers. Opt. Express 14, 13095-13100 (2006).

219. Tao, S. H. et al. Fractional optical vortex beam induced rotation of particles, Opt. Express 13, 7726-7731 (2005).

220. Gong, L. et al. Optical forces of focused femtosecond laser pulses on nonlinear optical Rayleigh particles. Photonics Res. 6, 138-143 (2018).

221. Zhang, Y. Q. et al. Nonlinearity-induced multiplexed optical trapping and manipulation with femtosecond vector beams. Nano Lett. 18, 5538-5543 (2018).

222. Shen, Z. et al. Visualizing orbital angular momentum of plasmonic vortices, Opt. Lett. 37, 4627-4629 (2012).

223. Zhang, Y. Q. et al. A plasmonic spanner for metal particle manipulation. Sci. Rep. 5, 15446 (2015).

224. Richardson, D. J., Fini, J. M. \& Nelson, L. E. Space-division multiplexing in optical fibres. Nat. Photonics 7, 354-362 (2013).

225. Wang, J. Advances in communications using optical vortices. Photonics Res. 4 , B14-B28 (2016). 
226. Willner, A. E. et al. Optical communications using orbital angular momentum beams. Adv. Opt. Photonics 7, 66-106 (2015).

227. Lavery, M. P. J. et al. Free-space propagation of high-dimensional structured optical fields in an urban environment. Sci. Adv. 3, e1700552 (2017).

228. Li, L. et al. High-capacity free-space optical communications between a ground transmitter and a ground receiver via a UAV using multiplexing of multiple orbital-angular-momentum beams. Sci. Rep. 7, 17427 (2017).

229. Yan, Y. et al. High-capacity millimetre-wave communications with orbital angular momentum multiplexing. Nat. Commun. 5, 4876 (2014).

230. Jia, P. et al. Sidelobe-modulated optical vortices for free-space communication. Opt. Lett. 38, 588-590 (2013).

231. Anguita, J. A., Herreros, J. \& Cisternas, J. E. Generation and detection of multiple coaxial vortex beams for free-space optical communications. In Proc. Quantum Electronics and Laser Science Conference. (Optical Society of America, San Jose, California, United States, 2012).

232. Heng, X. B. et al. All-fiber stable orbital angular momentum beam generation and propagation. Opt. Express 26, 17429-17436 (2018).

233. Xie, Z. W. et al. Integrated (de)multiplexer for orbital angular momentum fiber communication. Photonics Res. 6, 743-749 (2018).

234. Lei, T. et al. Massive individual orbital angular momentum channels for multiplexing enabled by Dammann gratings. Light Sci. Appl. 4, e257 (2015).

235. Ren, Y. X. et al. Orbital angular momentum-based space division multiplexing for high-capacity underwater optical communications. Sci. Rep. 6, 33306 (2016).

236. D'Ambrosio, V. et al. Complete experimental toolbox for alignment-free quantum communication. Nat. Commun. 3, 961 (2012).

237. Zhu, L. et al. Orbital angular momentum mode groups multiplexing transmission over 2.6-km conventional multi-mode fiber. Opt. Express 25, 25637-25645 (2017).

238. Mafu, M. et al. Higher-dimensional orbital-angular-momentum-based quantum key distribution with mutually unbiased bases. Phys. Rev. A 88, 032305 (2013).

239. Sit, A. et al. High-dimensional intracity quantum cryptography with structured photons. Optica 4, 1006-1010 (2017).

240. Nicolas, A. et al. A quantum memory for orbital angular momentum photonic qubits. Nat. Photonics 8, 234-238 (2014).

241. Ding, D. S. et al. Quantum storage of orbital angular momentum entanglement in an atomic ensemble. Phys. Rev. Lett. 114, 050502 (2015).

242. Zhou, Z. Q. et al. Quantum storage of three-dimensional orbital-angularmomentum entanglement in a crystal. Phys. Rev. Lett. 115, 070502 (2015).

243. Chen, L. X., Lei, J. J. \& Romero, J. Quantum digital spiral imaging. Light Sci. Appl. 3, e153 (2014).

244. Nagali, E. et al. Optimal quantum cloning of orbital angular momentum photon qubits through Hong-Ou-Mandel coalescence. Nat. Photonics 3, 720-723 (2009).

245. Ndagano, B. et al. Creation and detection of vector vortex modes for classical and quantum communication. J. Lightwave Technol. 36, 292-301 (2018).

246. Wang, X. L. et al. Quantum teleportation of multiple degrees of freedom of a single photon. Nature 518, 516-519 (2015).

247. Wang, X. L. et al. 18-qubit entanglement with six photons' three degrees of freedom. Phys. Rev. Lett. 120, 260502 (2018).

248. Toninelli, E. et al. Concepts in quantum state tomography and classical implementation with intense light: a tutorial. Adv. Opt. Photonics 11, 67-134 (2019).

249. Sephton, B. et al. A versatile quantum walk resonator with bright classical light. PLOS ONE 14, e0214891 (2019).

250. Ndagano, B. et al. Characterizing quantum channels with non-separable states of classical light. Nat. Phys. 13, 397-402 (2017).

251. Vieira, J. et al. Amplification and generation of ultra-intense twisted laser pulses via stimulated Raman scattering. Nat. Commun. 7, 10371 (2016).

252. Lenzini, F. et al. Optical vortex interaction and generation via nonlinear wave mixing. Phys. Rev. A 84, 061801 (2011).

253. Jiang, W. et al. Computation of topological charges of optical vortices via nondegenerate four-wave mixing. Phys. Rev. A 74, 043811 (2006).

254. Li, G. X. et al. Continuous control of the nonlinearity phase for harmonic generations. Nat. Mater. 14, 607-612 (2015).

255. Tymchenko, M. et al. Gradient nonlinear Pancharatnam-Berry metasurfaces. Phys. Rev. Lett. 115, 207403 (2015).

256. Keren-Zur, S. et al. Nonlinear beam shaping with plasmonic metasurfaces. ACS Photonics 3, 117-123 (2015).
257. Li, G. X., Zentgraf, T. \& Zhang, S. Rotational Doppler effect in nonlinear optics. Nat. Phys. 12, 736-740 (2016).

258. Musarra, G. et al. Rotation-dependent nonlinear absorption of orbital angular momentum beams in ruby. Opt. Lett. 43, 3073-3075 (2018).

259. Qiu, C. W. \& Yang, Y. J. Vortex generation reaches a new plateau. Science 357, 645 (2017).

260. Toda, Y. et al. Single orbital angular mode emission from externally feedbacked vertical cavity surface emitting laser. Appl. Phys. Lett. 111, 101102 (2017).

261. Miao, P. et al. Orbital angular momentum microlaser. Science 353, 464-467 (2016).

262. Wang, J. Metasurfaces enabling structured light manipulation: advances and perspectives [Invited]. Chin. Opt. Lett. 16, 050006 (2018).

263. Huang, L. L. et al. Dispersionless phase discontinuities for controlling light propagation. Nano Lett. 12, 5750-5755 (2012).

264. Sun, Y. Z. et al. Vector beam generation via micrometer-scale photonic integrated circuits and plasmonic Nano-antennae. J. Opt. Soc. Am. B 33, 360-366 (2016).

265. Chen, P. et al. Digitalizing self-assembled chiral superstructures for optical vortex processing. Adv. Mater. 30, 1705865 (2018).

266. Chen, Y. et al. Mapping twisted light into and out of a photonic chip. Phys. Rev. Lett. 121, 233602 (2018).

267. Jin, Y. et al. Dynamic modulation of spatially structured polarization fields for real-time control of ultrafast laser-material interactions. Opt. Express 21, 25333-25343 (2013).

268. Allegre, O. J. et al. Complete wavefront and polarization control for ultrashortpulse laser microprocessing. Opt. Express 21, 21198-21207 (2013).

269. Toyoda, K. et al. Transfer of light helicity to nanostructures. Phys. Rev. Lett. 110, 143603 (2013).

270. Syubaev, S. et al. Direct laser printing of chiral plasmonic nanojets by vortex beams. Opt. Express 25, 10214-10223 (2017).

271. Masuda, K. et al. Azo-polymer film twisted to form a helical surface relief by illumination with a circularly polarized Gaussian beam. Opt. Express 25, 12499-12507 (2017).

272. Takahashi, F. et al. Picosecond optical vortex pulse illumination forms a monocrystalline silicon needle. Sci. Rep. 6, 21738 (2016).

273. Torner, L., Torres, J. P. \& Carrasco, S. Digital spiral imaging. Opt. Express 13, 873-881 (2005).

274. Tan, P. S. et al. High-resolution wide-field standing-wave surface plasmon resonance fluorescence microscopy with optical vortices. Appl. Phys. Lett. 97, 241109 (2010).

275. Zhang, C. L. et al. Perfect optical vortex enhanced surface plasmon excitation for plasmonic structured illumination microscopy imaging. Appl. Phys. Lett. 108, 201601 (2016).

276. Xie, X. S. et al. Harnessing the point-spread function for high-resolution farfield optical microscopy. Phys. Rev. Lett. 113, 263901 (2014).

277. Wei, S. B. et al. Sub-100nm resolution PSIM by utilizing modified optical vortices with fractional topological charges for precise phase shifting. Opt. Express 23, 30143-30148 (2015).

278. Kozawa, Y., Matsunaga, D. \& Sato, S. Superresolution imaging via superoscillation focusing of a radially polarized beam. Optica 5, 86-92 (2018).

279. Willig, K. I. et al. STED microscopy reveals that synaptotagmin remains clustered after synaptic vesicle exocytosis. Nature 440, 935-939 (2006).

280. Wang, S. B. \& Chan, C. T. Lateral optical force on chiral particles near a surface. Nat. Commun. 5, 3307 (2014).

281. Brullot, W. et al. Resolving enantiomers using the optical angular momentum of twisted light. Sci. Adv. 2, e1501349 (2016).

282. Zhao, Y. et al. Chirality detection of enantiomers using twisted optical metamaterials. Nat. Commun. 8, 14180 (2017).

283. Jeffries, G. D. M. et al. Using polarization-shaped optical vortex traps for single-cell nanosurgery. Nano Lett. 7, 415-420 (2007).

284. Stellinga, D. et al. An organic vortex laser. ACS Nano 12, 2389-2394 (2018),

285. Lavery, M. P. J. et al. Detection of a spinning object using light's orbital angular momentum. Science 341, 537-540 (2013).

286. Cvijetic, N. et al. Detecting lateral motion using light's orbital angular momentum. Sci. Rep. 5, 15422 (2015).

287. Kravets, V. G. et al. Singular phase nano-optics in plasmonic metamaterials for label-free single-molecule detection. Nat. Mater. 12, 304-309 (2013).

288. Xie, G. D. et al. Using a complex optical orbital-angular-momentum spectrum to measure object parameters. Opt. Lett. 42, 4482-4485 (2017). 
289. Fu, S. Y. \& Gao, C. Q. Influences of atmospheric turbulence effects on the orbital angular momentum spectra of vortex beams. Photonics Res. 4, B1-B4 (2016).

290. Li, Y., Yu, L. \& Zhang, Y. X. Influence of anisotropic turbulence on the orbital angular momentum modes of Hermite-Gaussian vortex beam in the ocean. Opt. Express 25, 12203-12215 (2017)

291. Min, C. J. et al. Plasmonic nano-slits assisted polarization selective detour phase meta-hologram. Laser Photonics Rev. 10, 978-985 (2016).

292. Xie, Z. W. et al. On-chip spin-controlled orbital angular momentum directional coupling. J. Phys. D Appl. Phys. 51, 014002 (2017).

293. Zhang, C. \& Ma, L. Detecting the orbital angular momentum of electro-magnetic waves using virtual rotational antenna. Sci. Rep. 7, 4585 (2017).

294. Mei, S. T. et al. On-chip discrimination of orbital angular momentum of light with plasmonic nanoslits. Nanoscale 8, 2227-2233 (2016).

295. Foo, G., Palacios, D. M. \& Swartzlander, G. A. Optical vortex coronagraph. Opt. Lett. 30, 3308-3310 (2005).

296. Lee, J. H. et al. Experimental verification of an optical vortex coronagraph. Phys. Rev. Lett. 97, 053901 (2006).

297. Swartzlander, G. A. et al. Astronomical demonstration of an optical vortex coronagraph. Opt. Express 16, 10200-10207 (2008).
298. Mawet, D. et al. Annular groove phase mask coronagraph. Astrophys. J. 633 1191-1200 (2005).

299. Absil, O. et al. Three years of harvest with the vector vortex coronagraph in the thermal infrared. In Proc. SPIE 9908, Ground-based and Airborne Instrumentation for Astronomy VI. 99080 Q (SPIE, Edinburgh, United Kingdom, 2016).

300. Aleksanyan, A., Kravets, N. \& Brasselet, E. Multiple-star system adaptive vortex coronagraphy using a liquid crystal light valve. Phys. Rev. Lett. 118, 203902 (2017).

301. Aleksanyan, A. \& Brasselet, E. High-charge and multiple-star vortex coronagraphy from stacked vector vortex phase masks. Opt. Lett. 43, 383-386 (2018).

302. Tamburini, F. et al. Twisting of light around rotating black holes. Nat. Phys. 7, 195-197 (2011).

303. Battersby, S. Twisting the light away. New Sci. 182, 36-40 (2004).

304. Yin, J. Y. et al. Microwave vortex-beam emitter based on spoof surface plasmon polaritons. Laser Photonics Rev. 12, 1600316 (2018).

305. Marzo, A., Caleap, M. \& Drinkwater, B. W. Acoustic virtual vortices with tunable orbital angular momentum for trapping of Mie particles. Phys. Rev. Lett. 120, 044301 (2018).

306. Bialynicki-Birula, I. \& Charzyński, S. Trapping and guiding bodies by gravitational waves endowed with angular momentum. Phys. Rev. Lett. 121, 171101 (2018). 